Article

\title{
Photodegradation Study of Sertindole by UHPLC-ESI-Q-TOF and Influence of Some Metal Oxide Excipients on the Degradation Process
}

\author{
Jakub Trawiński ${ }^{D}$ and Robert Skibiński * (D) \\ Department of Medicinal Chemistry, Faculty of Pharmacy, Medical University of Lublin, Jaczewskiego 4, \\ 20-090 Lublin, Poland \\ * Correspondence: robert.skibinski@umlub.pl; Tel.: +48-81-4487383; Fax: +48-81-4487381
}

Received: 23 May 2019; Accepted: 25 June 2019; Published: 27 June 2019

check for updates

\begin{abstract}
The evaluation of the influence of the excipients present in the pharmaceutical formulations on the drug stability is an important part of quality control of medicines. One of the most commonly applied group of excipients are pigments, such as titanium dioxide or various forms of iron oxides, which are well-known photocatalytic agents. Therefore, the photostability of an atypical antipsychotic drug sertindole and the influence of pigments commonly used in the pharmaceutical formulations $\left(\mathrm{FeOOH}, \mathrm{Fe}_{2} \mathrm{O}_{3}\right.$, and $\mathrm{TiO}_{2}$ ) on this process were studied. The quantitative and qualitative analysis of the process was performed with the use of ultra high pressure liquid chromatography with diode array detection (UHPLC-DAD) system coupled with a high resolution hybrid electrospray ionization quadrupole time-of-flight (ESI-Q-TOF) mass spectrometer. Sertindole turned out to be a highly photolabile molecule. Overall 18 transformation products were found, mainly formed as a consequence of dechlorination, hydroxylation, and dehydrogenation. In all the experiments, except the $\mathrm{TiO}_{2}$-mediated photocatalysis, the product of chlorine substitution with a hydroxyl group was the major product. The presence of $\mathrm{Fe}_{2} \mathrm{O}_{3}$ and $\mathrm{TiO}_{2}$ accelerated the degradation process, while $\mathrm{FeOOH}$ served as its inhibitor. The experiments conducted with the use of the pharmaceutical formulations confirmed the catalytic activity of the used excipients. The exploration of the obtained phototransformation profiles with the use of principal component analysis (PCA) revealed that the presence of both iron oxides could influence the qualitative and quantitative aspect of the studied processes. In silico assessment of the properties showed that the transformation products are generally less toxic to rodents, possess lower hERG blocking potential, but could be more mutagenic than the parent molecule.
\end{abstract}

Keywords: sertindole; iron oxides; titanium dioxide; photodegradation; photocatalysis; PCA

\section{Introduction}

Nowadays, photostability testing is an indispensable part of the drug analysis. As stated in the European Pharmacopoeia, over 250 active pharmaceutical substances (APIs) are classified as photolabile molecules and their exposure to light should be avoided [1]. The consequences of such behavior could be divided into two groups. First, the interaction of APIs with UV-Vis radiation (natural or artificial) leads to their decomposition which involves the decrease or loss of the therapeutic efficiency. Second, the phototransformation products (TPs) can possess their own pharmacological activity or toxic properties significantly higher than the parent compound [2]. For that reason various guidelines regarding the photostability testing of drugs were implemented. One of them was proposed by the International Council for Harmonization of Technical Requirements for Pharmaceuticals for Human Use (ICH) as the "Stability Testing: Photostability Testing of New Drug Substances and Products" (Q1B) document [3]. 
The influence of metal oxides should be important from a technological point of view, relative to stability and safety, and also from an analytical point of view. One of the most commonly applied group of excipients are pigments such as titanium dioxide (white pigment) or various forms of iron oxides (yellow or red pigments). Taking into account the commonly known photocatalytic properties of such compounds [4], the assessment of their impact on the photodegradation process is particularly interesting. Although the effect of various forms of $\mathrm{TiO}_{2}$ addition to the pharmaceutical formulations was studied [5], the influence of the other pigmenting substances seems to be still unexplored.

Sertindole (1-[2-[4-[5-chloro-1-(4-fluorophenyl)-indol-3-yl]piperidin-1-yl]ethyl]-imidazolidin-2-one) is an atypical antipsychotic drug used for the treatment of schizophrenia and is effective both against the positive and negative symptoms of the disease [6]. The drug serves as a 5-HT2 ${ }_{\mathrm{A}}, 5-\mathrm{HT} 2_{\mathrm{C}}, \mathrm{D}_{2}$, and $\alpha_{1}$ receptors antagonist and has a selective influence on the mesolimbic dopamine neurons [7]. Such pharmacological properties are responsible for fewer extrapyramidal side effects than in the case of classical antipsychotics [8], but they can prolong the QT interval on electrocardiogram [9]. In the available literature data there are several papers describing the determination of sertindole as well as its metabolites both in the pharmaceutical formulations and biological samples using various analytical methods such as high performance liquid chromatography with ultraviolet detection (HPLC-UV) [10], fluorimetrics [11,12], or high performance liquid chromatography with mass spectrometry detection (HPLC-MS) [13]. One research paper reporting the stability-indicating methods with the use of HPLC and TLC-densitometry was also published [14], but only one TP was found and structurally characterized. Moreover, on the contrary to our observations, in the above study sertindole was found to be totally stable under the photolytic stress conditions.

The aim of the following work is to study the direct photolysis of sertindole under the simulated solar radiation, the determination of the photolysis kinetics, the identification of the TPs, in silico evaluation of toxicity, and chemometric analysis of the obtained results. Additionally, in order to assess the influence of pigment excipients on the phototransformation process, the effect of addition of titanium dioxide and two forms of iron oxide (yellow and red) was also evaluated. The obtained results were compared with the experiments conducted on the real sertindole pharmaceutical formulations-one containing $\mathrm{TiO}_{2}$ and yellow iron oxide and the other one also containing $\mathrm{TiO}_{2}$ and red iron oxide.

\section{Experimental}

\subsection{Materials}

Serdolect ${ }^{\circledR} 4 \mathrm{mg}$ and $16 \mathrm{mg}$ tablets (H. Lundbeck A/S, Valby, Denmark), containing $4 \mathrm{mg}$ and $16 \mathrm{mg}$ of sertindole in each tablet, respectively, were obtained from the local pharmacy. Sertindole standard, water for liquid chromatography-mass spectrometry (LC-MS), acetonitrile for LC-MS, formic acid for LC-MS, and titanium (IV) oxide, nanopowder $21 \mathrm{~nm}$ particle size (Aeroxide ${ }^{\circledR}$ 25) were purchased from Sigma Aldrich Co. (St. Louis, MO, USA). Water gradient grade for liquid chromatography were purchased from Merck (Darmstadt, Germany). Yellow iron oxide nanorods (FeOOH alpha, 98\% $50 \mathrm{~nm} \times 10 \mathrm{~nm})$ and red iron oxide $\left(\mathrm{Fe}_{2} \mathrm{O}_{3}\right.$, alpha, 98+\%, 20-40 nm) were purchased from US Research Nanomaterials, Inc. (Houston, TX, USA).

\subsection{Sample Preparation}

The stock solution of sertindole was prepared in acetonitrile at concentration $0.5 \mathrm{mg} \mathrm{mL}^{-1}$ and was refrigerated at $7{ }^{\circ} \mathrm{C}$. The working solutions were prepared by diluting the stock solution in water to obtain $10 \mu \mathrm{g} \mathrm{mL}^{-1}$. The catalytic suspensions were prepared by weighing an appropriate amount of each catalyst $\left(\mathrm{TiO}_{2}, \mathrm{Fe}_{2} \mathrm{O}_{3}\right.$, and $\left.\mathrm{FeOOH}\right)$ or a mixture of catalysts $\left(\mathrm{TiO}_{2}-\mathrm{Fe}_{2} \mathrm{O}_{3}\right.$ and $\left.\mathrm{TiO}_{2}-\mathrm{FeOOH}\right)$ in the volumetric flasks and adding the sertindole working solution. The obtained catalysts and catalysts mixtures loading was $100 \mu \mathrm{g} \mathrm{mL}^{-1}$ (in the case of mixtures $50 \mu \mathrm{g} \mathrm{mL}^{-1}$ the loading of each catalyst was used). The suspensions containing pharmaceutical formulations were prepared as follows: first, Serdolect ${ }^{\circledR} 4 \mathrm{mg}$ and $16 \mathrm{mg}$ tablets were separately grounded in a mortar and the equivalents of $250 \mu \mathrm{g}$ 
of sertindole were weighed and transferred into $25 \mathrm{~mL}$ volumetric flasks. Then $500 \mu \mathrm{L}$ of the LC-MS acetonitrile was added to the flasks and after $5 \mathrm{~min}$ of ultrasonic sweeping the flasks were filled with ultrapure water. The obtained concentrations of sertindole and acetonitrile were on the same level as in the case of a standard solution.

\subsection{Irradiation Procedure}

For all the experiments the working solutions and suspensions were transferred into $3.5 \mathrm{~mL}$ quartz caped cells $(1=1 \mathrm{~cm})$ mounted horizontally in Atlas Suntest CPS+ photostability chamber (Linsengericht, Germany), and irradiated simultaneously. The irradiance was set to $250 \mathrm{~W} \mathrm{~m}^{-2}$ which corresponds to energy dose of $900 \mathrm{~kJ} \mathrm{~m}^{-2} \mathrm{~h}^{-1}$. The chamber was equipped with a xenon lamp and D65 filter simulating full solar spectrum. The temperature in the chamber was controlled and kept below $35{ }^{\circ} \mathrm{C}$. All the suspensions were vigorously stirred (500 rpm) with the use of a microstirrer (MINI Stirrer, Cimarel: Telemodul, Thermo Electron LED GmbH, Langenselbold, Germany) and polytetrafluoroethylene (PTFE) covered stirring bar $(1=6 \mathrm{~mm})$ during the whole experiment. The dark control sample was also performed by exposing the working solution in quartz cell wrapped in aluminum foil for the same period of time. $100 \mu \mathrm{L}$ solution or suspension aliquots were collected after $0,2,4,8,12$, and $14 \mathrm{~min}$ and then centrifuged at 15,000 rpm for $5 \mathrm{~min}$. The UHPLC-DAD/ESI-Q-TOF analysis was performed afterward.

\subsection{Analytical Procedure}

UHPLC-MS/MS analysis was performed using the Agilent Accurate-Mass Q-TOF LC/MS G6520B system with dual electrospray source and Infinity 1290 UHPLC system consisting of: binary pump G4220A, FC/ALS thermostat G1330B, autosampler G4226A, DAD detector G4212A, TCC G1316C module (Agilent Technologies, Santa Clara, CA, USA), and Hibar RP-18e $(2.1 \times 50 \mathrm{~mm}, \mathrm{dp}=2 \mu \mathrm{m}) \mathrm{HR}$ column (Merck, Darmstadt, Germany). A mixture of acetonitrile (A) and water containing $0.1 \%$ of formic acid (B) was used as a mobile phase. The gradient elution was carried out at a constant flow of $0.3 \mathrm{~mL} \mathrm{~min}^{-1}$ from 5\% A (95\% B) to 60\% 0-9 min. Two-minute post time was performed to return to initial conditions. The total analysis time was $11 \mathrm{~min}$. The injection volume was $1 \mu \mathrm{L}$ and the column temperature was maintained at $35^{\circ} \mathrm{C}$. MassHunter workstation software in version B.08.00 was used for the control of the system and data acquisition.

The MS detector was tuned in a positive mode in extended dynamic range ( $2 \mathrm{GHz}$ ). To ensure accuracy in masses measurements, reference mass correction was also used and mass 121.0508 and 922.0097 (Agilent ES TOF reference mix solution) were used as lock masses. The electrospray (ESI) was chosen as an ion source. The main parameters were optimized and the following settings were applied: gas temp.: $300^{\circ} \mathrm{C}$, drying gas: $9 \mathrm{~L} / \mathrm{min}$, nebulizer pressure: 30 psig, capillary voltage: $3500 \mathrm{~V}$, fragmentor voltage: $175 \mathrm{~V}$, skimmer voltage: $65 \mathrm{~V}$, octopole $1 \mathrm{RF}$ voltage: $750 \mathrm{~V}$. In order to make the qualitative and quantitative analysis in one run, data acquisition was performed in auto MS/MS mode with spectral parameters: mass range: $60-950 \mathrm{~m} / \mathrm{z}$ and acquisition rate: 2 spectra/s (for MS and MS/MS data). Diode array detector collected data in the range $210-300 \mathrm{~nm}$, and wavelength of $260 \mathrm{~nm}$ was selected for the quantitative analysis of sertindole. All the measurements were duplicated. For the chemometric analysis, the data acquisition was performed in the TOF mode, and six replications were done for each experiment.

Calibration of the quantitative method was performed with the use of six sertindole concentration levels $\left(0.5,1,5,10,15\right.$, and $\left.20 \mu \mathrm{g} \mathrm{mL}^{-1}\right)$. Intraday and interday precision was evaluated using $n=12$ and $n=18$ replicates. The averaged retention time of sertindole was $7.103 \pm 0.006 \mathrm{~min}$. All that data, along with the limits of detection and quantification (LOD and LOQ) are shown in Table 1. 
Table 1. Validation results of the developed method with diode array detection (DAD) detection.

\begin{tabular}{|c|c|}
\hline Parameters & Results \\
\hline \multicolumn{2}{|l|}{ Linearity } \\
\hline Concentration range $\left(\mu \mathrm{g} \mathrm{mL}^{-1}\right)$ & $(0.5: 20)$ \\
\hline Slope & 4.5894 \\
\hline $\mathrm{SD}^{\mathrm{a}}$ of slope & 0.1143 \\
\hline Intercept & 0.4528 \\
\hline $\mathrm{SD}^{\mathrm{a}}$ of intercept & 0.1809 \\
\hline Correlation coefficient $(r)$ & 0.9998 \\
\hline $\mathrm{LOD}^{\mathrm{b}}\left(\mu \mathrm{g} \mathrm{mL} \mathrm{g}^{-1}\right)$ & 0.034 \\
\hline $\mathrm{LOQ}^{\mathrm{c}}(\mu \mathrm{g} \mathrm{mL}-1)$ & 0.100 \\
\hline \multicolumn{2}{|l|}{ Precision (RSD ${ }^{\mathrm{d}} \%$ ) } \\
\hline Intra-day $(n=12)$ & 1.74 \\
\hline Inter-day $(n=18)$ & 2.53 \\
\hline \multicolumn{2}{|l|}{ Accuracy } \\
\hline Recovery (\%) & 100.20 \\
\hline $\operatorname{RSD}^{\mathrm{d}}(\%)$ & 2.77 \\
\hline
\end{tabular}

\subsection{Chemometric Analysis}

Six samples taken after 4 min of irradiation from each experiment were taken and submitted for LC-MS analysis. TOF (MS) mode was used for the registration of their chromatographic/spectral degradation profiles. The MFE (molecular feature extraction) algorithm from the Mass Hunter Qualitative Analysis software version B.06.00 (Agilent) was used for the data background ion noise cleaning and to extract the list of the ions characteristic for photocatalytic transformation products. The MFE parameters were optimized and the following settings were applied: maximum 1 charge state of the analyzed ions, more than 5000 counts for the compound filter, isotope model: common organic molecules with peak spacing tolerance $0.0025 \mathrm{~m} / \mathrm{z}$.

In order to carry out the multivariate chemometric analysis, the obtained results were next exported to the Mass Profiler Professional (MPP) software version 12.61 (Agilent Technologies Inc., Santa Clara, CA, USA and Strand Life Sciences Pvt. Ltd., Bengaluru, India). With the use of this software the data was normalized and aligned. Then the principal component analysis (PCA) was performed in order to evaluate the qualitative and quantitative differences in the registered phototransformation profiles.

\subsection{In Silico Evaluation of Sertindole and its TPs Properties}

The acute toxicity to rodents, mutagenicity and hERG inhibitory potential of the identified phototransformation products as well as of the parent compound were calculated using the ACD/Percepta 14.0.0 (ACD/Labs, 2015 Release) software. Then the multivariate chemometric analysis (PCA) was performed in order to compare the toxicity of the photoproducts and the toxicity assessment methods. Data preprocessing and PCA analysis were performed using the R 3.2.3 software (GNU project). The obtained data was centered and scaled before the chemometric analysis.

\section{Results and Discussion}

\subsection{Optimization of the LC-ESI-MS/MS Method}

The chromatographic conditions were optimized based on the UHPLC reversed-phase C18 column and various organic modifiers, buffers, and elution systems including gradient elution were tested. Finally, the gradient elution with a mixture of acetonitrile and water with addition of $0.1 \%$ of formic acid as a mobile phase was chosen.

The choice between the two most frequently used in LC-MS ionization methods, electrospray ionization (ESI) and atmospheric pressure chemical ionization (APCI), was made on the basis of the 
preliminary study and the comparison of these sources for the investigation of phototransformation process of several psychotropic drugs including sertindole. According to this research, ESI outperformed APCI [15].

\subsection{Quantitative Study of the Phototransformation Process}

\subsubsection{Calibration and Validation of the Method}

The calibration curve was linear over the concentration range $\left(0.5-20 \mu \mathrm{gL}^{-1}\right)$, and satisfied the following equation: $y=4.5894( \pm 0.01143) x+0.45228( \pm 0.1809)$ (where $y$ is sertindole concentration and $x$ stands for a peak area) with the correlation coefficient 0.9998 . The complete data concerning a quantitative method calibration including inter- and intra-day precision, LOD, and LOQ is presented in Table 1 and Table S1. The obtained equation was then used to calculate sertindole concentration during the photodegradation experiments. Robustness of the method was also tested and the obtained results are presented in Table S2.

\subsubsection{Phototransformation Kinetics}

In all the studied cases the photodecomposition reaction fitted the pseudo-first order model $\left(\ln \mathrm{c}=\ln \mathrm{c}_{0}-\mathrm{kt}\right)$. Half-life of sertindole during the direct photolysis process was almost the highest amongst all the conducted experiments and amounted to $6.96 \mathrm{~min}$. Only in the FeOOH-mediated photocatalysis half-life was higher $(8.36 \mathrm{~min})$. All the remaining photocatalytic processes as well as experiments with pharmaceutical formulations resulted in faster decomposition of the parent compound (Figure 1). As it was expected, the lowest half-life was observed in the $\mathrm{TiO}_{2}$-mediated photocatalysis $(1.67 \mathrm{~min})$. The summary of the sertindole photodecomposition kinetics is presented in Table 2.

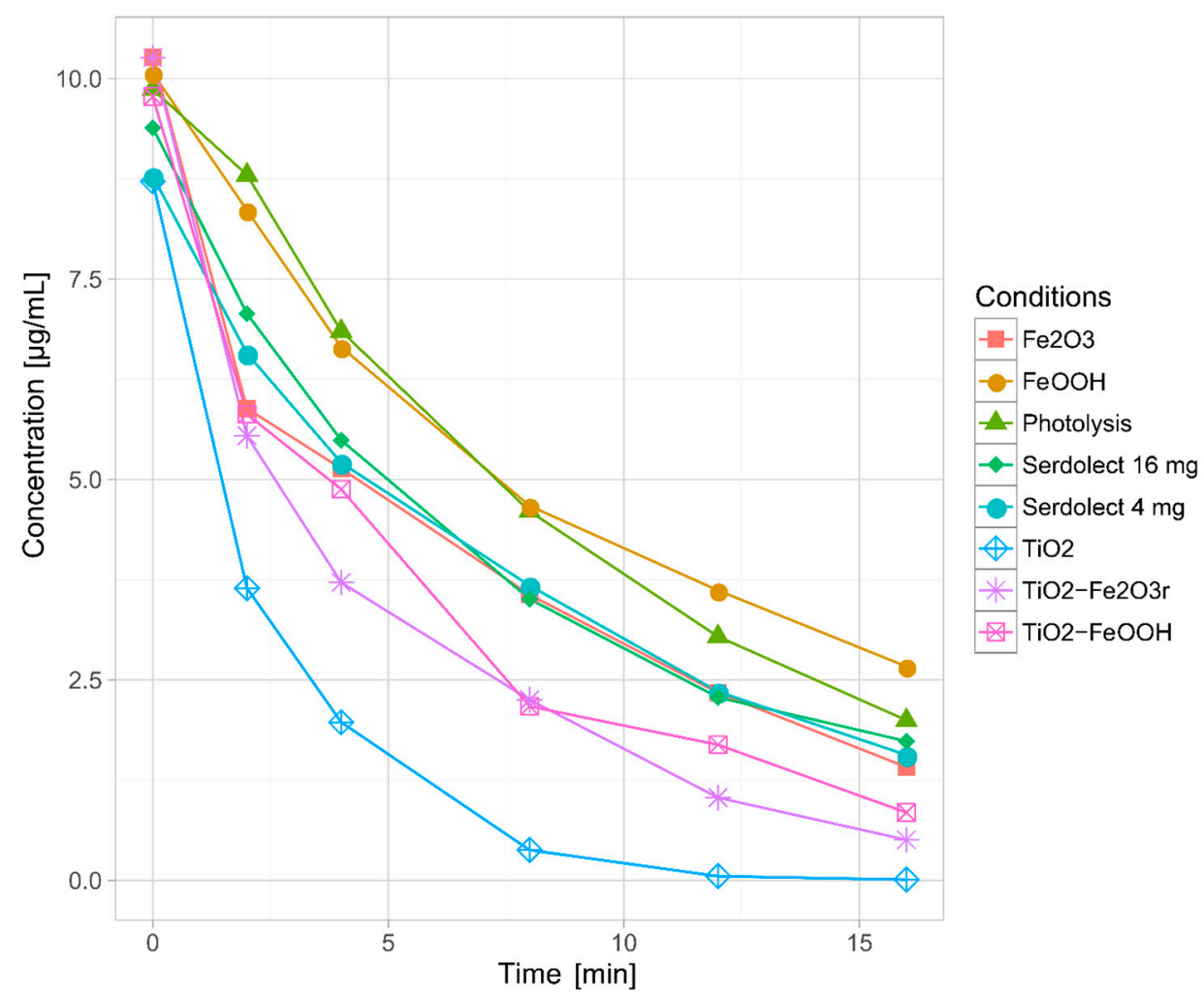

Figure 1. Kinetics of sertindole photodecomposition. 
Table 2. Summary of sertindole degradation kinetics parameters.

\begin{tabular}{cllll}
\hline Experiment & \multicolumn{1}{c}{ Model } & Fit $(\boldsymbol{r})$ & $k \pm S D\left(\mathbf{m i n}^{-\mathbf{1}}\right)$ & $\boldsymbol{t}_{\mathbf{1 / 2}}(\mathbf{m i n})$ \\
\hline Direct photolysis & Pseudo-first-order & 0.9989 & $0.0996 \pm 5.22 \times 10^{-6}$ & 6.96 \\
$\mathrm{TiO}_{2}$ & Pseudo-first-order & 0.9989 & $0.4143 \pm 3.42 \times 10^{-3}$ & 1.67 \\
$\mathrm{FeOOH}$ yellow & Pseudo-first-order & 0.9963 & $0.0829 \pm 8.13 \times 10^{-4}$ & 8.36 \\
$\mathrm{Fe}_{2} \mathrm{O}_{3}$ red & Pseudo-first-order & 0.9874 & $0.1236 \pm 8.96 \times 10^{-4}$ & 5.61 \\
$\mathrm{TiO}_{2}-\mathrm{FeOOH}_{\text {(yellow) }}$ & Pseudo-first-order & 0.9894 & $0.1524 \pm 9.64 \times 10^{-5}$ & 4.55 \\
$\mathrm{TiO}_{2}-\mathrm{Fe}_{2} \mathrm{O}_{3}$ (red) & Pseudo-first-order & 0.9950 & $0.1871 \pm 8.61 \times 10^{-4}$ & 3.70 \\
Serdolect 4 mg & Pseudo-first-order & 0.9984 & $0.1076 \pm 2.60 \times 10^{-3}$ & 6.44 \\
Serdolect $16 \mathrm{mg}$ & Pseudo-first-order & 0.9948 & $0.1052 \pm 1.61 \times 10^{-3}$ & 6.59 \\
\hline
\end{tabular}

Taking into account the obtained results, sertindole should be considered as a highly photolabile compound and should be protected from light. Unsurprisingly, the addition of the photocatalysts generally accelerated the degradation of the parent compound. The most spectacular results were achieved by adding $\mathrm{TiO}_{2}$, which was expected, as this compound is generally considered to be the most effective amongst the photocatalysts [4]. After 16 min of irradiation, sertindole was almost undetectable. On the other hand, the application of $\mathrm{FeOOH}$ resulted in the slowest degradation rate-even slower than in the case of direct photolysis. This phenomenon could be explained by a very low photocatalytic efficiency combined with the shielding effect-the presence of the catalyst increases the turbidity of the sample (which decreases the penetration of radiation), and weak photocatalytic activity is not capable to compensate such an effect. The application of $\mathrm{Fe}_{2} \mathrm{O}_{3}$ noticeably increased the degradation rate in comparison with the direct photolysis and was significantly more active than $\mathrm{FeOOH}$, which is consistent with previous studies [16]. Sertindole half-lives obtained in the experiments with the use of photocatalysts mixtures corresponded with the aforementioned results-the mixture of $\mathrm{TiO}_{2}$ and $\mathrm{Fe}_{2} \mathrm{O}_{3}$ catalyzed the photodecomposition more efficiently than $\mathrm{TiO}_{2}-\mathrm{FeOOH}$ mixture. The results of the pharmaceutical formulations experiments were particularly interesting. Half-lives in both cases were slightly lower than in the direct photolysis, but the differences between Serdolect ${ }^{\circledR} 4 \mathrm{mg}$ (S4) and Serdolect ${ }^{\circledR} 16 \mathrm{mg}$ (S16) were rather insignificant. This finding suggests that in the S4 and S16, photocatalytic processes $\mathrm{TiO}_{2}$ played the main role (at least from the quantitative point of view) and the presence of iron oxides did not substantially alter the degradation rate. Additionally, it should be noted that despite the shielding effect caused by photocatalytic-inactive excipients, the decomposition of sertindole was faster than in the direct photolysis. This suggests that photocatalysts present in the pharmaceutical formulations could play a significant role in the phototransformation processes and their influence on the formation of the TPs should be also studied.

In order to examine the influence of hydrolysis or adsorption on the $\mathrm{TiO}_{2}, \mathrm{Fe}_{2} \mathrm{O}_{3}, \mathrm{FeOOH}$ particles or on the substances present in the pharmaceutical formulations of S4 and S16, the dark control samples were performed simultaneously. No significant decrease in sertindole concentration was observed in the direct photolysis, photocatalysis, or pharmaceutical formulations experiments, which indicates that both hydrolysis and adsorption did not play any role in the removal of the parent compound.

\subsection{Identification of the Transformation Products}

The qualitative analysis of the phototransformation process generally was done with the use of auto MS/MS mode, automatically selecting the precursor ions for the fragmentation. However, in some cases the acquisition mode was changed to the targeted MS/MS, as well as some MS/MS experiments were repeated with the use of various collision induced dissociation (CID) energy in order to obtain maximum information about the TPs structure. MS/MS spectra of sertindole and its TPs, along with the suggested fragmentation patterns, are shown in the supplementary material (Figure S1-S19).

Overall, eighteen TPs were formed in the studied phototransformation processes, and their structures were elucidated on the basis of the obtained MS/MS spectra (Table 3). Half of the identified TPs were formed during all the experiments, however, in some cases only at a trace level. The remaining TPs were absent at least in one sample. The evolution profiles of the TPs formed in the experiments are presented in Figures 2-6 (TPs detected at a trace level are not shown). 
Table 3. Accurate masses of sertindole phototransformation products.

\begin{tabular}{|c|c|c|c|c|c|c|c|c|c|c|c|c|c|c|c|c|}
\hline \multirow{2}{*}{ Cpd. } & \multirow{2}{*}{$\begin{array}{c}t_{R} \\
(\mathrm{~min})\end{array}$} & \multirow{2}{*}{$\begin{array}{l}\text { Elemental } \\
\text { Formula } \\
(\mathrm{M}+\mathrm{H})^{+}\end{array}$} & \multicolumn{2}{|c|}{ Mass $(m / z)$} & \multirow{2}{*}{$\begin{array}{l}\text { Error } \\
(\mathrm{ppm})\end{array}$} & \multirow{2}{*}{ DBE } & \multicolumn{2}{|c|}{ Fragmentation (MS/MS) } & \multicolumn{8}{|c|}{ Occurrence } \\
\hline & & & Exp. & Theo. & & & Mass $(m / z)$ & $\begin{array}{c}\text { Elemental } \\
\text { Formula }\end{array}$ & $\mathrm{DP}^{\mathrm{a}}$ & $\mathbf{T}^{\mathrm{b}}$ & $\mathrm{Fy}^{\mathrm{c}}$ & $\mathrm{Fr}^{\mathrm{d}}$ & TFy ${ }^{\mathbf{e}}$ & $\mathrm{TFr}^{\mathrm{f}}$ & $\mathrm{S} 4^{\mathrm{g}}$ & $\mathrm{S} 16^{\mathrm{h}}$ \\
\hline Sert & 7.06 & $\mathrm{C}_{24} \mathrm{H}_{27} \mathrm{ClFN}_{4} \mathrm{O}$ & 441.1858 & 441.1852 & 1.36 & 13 & $\begin{array}{l}355.1379 \\
329.1200 \\
298.0765 \\
270.0464 \\
196.1443 \\
168.1131 \\
142.0976 \\
113.0713 \\
99.0562 \\
70.0655\end{array}$ & $\begin{array}{c}\mathrm{C}_{21} \mathrm{H}_{21} \mathrm{ClFN}_{2} \\
\mathrm{C}_{19} \mathrm{H}_{19} \mathrm{ClFN}_{2} \\
\mathrm{C}_{18} \mathrm{H}_{14} \mathrm{ClFN} \\
\mathrm{C}_{16} \mathrm{H}_{10} \mathrm{ClFN} \\
\mathrm{C}_{10} \mathrm{H}_{18} \mathrm{~N}_{3} \mathrm{O} \\
\mathrm{C}_{8} \mathrm{H}_{14} \mathrm{~N}_{3} \mathrm{O} \\
\mathrm{C}_{6} \mathrm{H}_{12} \mathrm{~N}_{3} \mathrm{O} \\
\mathrm{C}_{5} \mathrm{H}_{9} \mathrm{~N}_{2} \mathrm{O} \\
\mathrm{C}_{4} \mathrm{H}_{7} \mathrm{~N}_{2} \mathrm{O} \\
\mathrm{C}_{4} \mathrm{H}_{8} \mathrm{~N}\end{array}$ & + & + & + & + & + & + & + & + \\
\hline TP1 & 5.50 & $\mathrm{C}_{24} \mathrm{H}_{28} \mathrm{FN}_{4} \mathrm{O}_{2}$ & 423.2194 & 423.2191 & 0.71 & 13 & $\begin{array}{c}337.1704 \\
311.1584 \\
266.0951 \\
196.1443 \\
168.1123 \\
142.0976 \\
113.0713 \\
84.0810 \\
70.0653\end{array}$ & $\begin{array}{c}\mathrm{C}_{21} \mathrm{H}_{22} \mathrm{FN}_{2} \mathrm{O} \\
\mathrm{C}_{19} \mathrm{H}_{20} \mathrm{FN}_{2} \mathrm{O} \\
\mathrm{C}_{17} \mathrm{H}_{13} \mathrm{FNO} \\
\mathrm{C}_{10} \mathrm{H}_{18} \mathrm{~N}_{3} \mathrm{O} \\
\mathrm{C}_{8} \mathrm{H}_{14} \mathrm{~N}_{3} \mathrm{O} \\
\mathrm{C}_{6} \mathrm{H}_{12} \mathrm{~N}_{3} \mathrm{O} \\
\mathrm{C}_{5} \mathrm{H}_{9} \mathrm{~N}_{2} \mathrm{O} \\
\mathrm{C}_{5} \mathrm{H}_{10} \mathrm{~N} \\
\mathrm{C}_{4} \mathrm{H}_{8} \mathrm{~N}\end{array}$ & + & + & + & + & + & + & + & + \\
\hline TP2 & 5.48 & $\mathrm{C}_{24} \mathrm{H}_{28} \mathrm{FN}_{4} \mathrm{O}_{4}$ & 455.2086 & 455.2089 & -0.66 & 13 & $\begin{array}{l}439.2115 \\
421.1996 \\
194.1250 \\
113.0712 \\
\end{array}$ & $\begin{array}{c}\mathrm{C}_{24} \mathrm{H}_{28} \mathrm{FN}_{4} \mathrm{O}_{3} \\
\mathrm{C}_{24} \mathrm{H}_{26} \mathrm{FN}_{4} \mathrm{O}_{2} \\
\mathrm{C}_{10} \mathrm{H}_{16} \mathrm{~N}_{3} \mathrm{O} \\
\mathrm{C}_{5} \mathrm{H}_{9} \mathrm{~N}_{2} \mathrm{O}\end{array}$ & + & + & + & + & + & + & + & + \\
\hline TP3 & 5.31 & $\mathrm{C}_{24} \mathrm{H}_{29} \mathrm{~N}_{4} \mathrm{O}_{2}$ & 405.2283 & 405.2285 & -0.49 & 13 & $\begin{array}{c}319.1767 \\
196.1445 \\
113.0712 \\
84.0807 \\
71.0622 \\
\end{array}$ & $\begin{array}{c}\mathrm{C}_{21} \mathrm{H}_{23} \mathrm{~N}_{2} \mathrm{O} \\
\mathrm{C}_{10} \mathrm{H}_{18} \mathrm{~N}_{3} \mathrm{O} \\
\mathrm{C}_{5} \mathrm{H}_{9} \mathrm{~N}_{2} \mathrm{O} \\
\mathrm{C}_{5} \mathrm{H}_{10} \mathrm{~N} \\
\mathrm{C}_{3} \mathrm{H}_{7} \mathrm{~N}_{2}\end{array}$ & + & - & + & + & $+^{*}$ & $+^{*}$ & + & + \\
\hline TP4 & 4.72 & $\mathrm{C}_{24} \mathrm{H}_{26} \mathrm{FN}_{4} \mathrm{O}_{3}$ & 437.1983 & 437.1983 & 0 & 14 & $\begin{array}{l}339.1500 \\
296.1094 \\
194.1286 \\
168.1123 \\
142.0950 \\
113.0705\end{array}$ & $\begin{array}{l}\mathrm{C}_{20} \mathrm{H}_{20} \mathrm{FN}_{2} \mathrm{O} \\
\mathrm{C}_{18} \mathrm{H}_{10} \mathrm{FNO}_{2} \\
\mathrm{C}_{10} \mathrm{H}_{16} \mathrm{~N}_{3} \mathrm{O} \\
\mathrm{C}_{8} \mathrm{H}_{14} \mathrm{~N}_{3} \mathrm{O} \\
\mathrm{C}_{6} \mathrm{H}_{12} \mathrm{~N}_{3} \mathrm{O} \\
\mathrm{C}_{5} \mathrm{H}_{9} \mathrm{~N}_{2} \mathrm{O}\end{array}$ & + & + & + & + & + & + & + & + \\
\hline
\end{tabular}


Table 3. Cont

\begin{tabular}{|c|c|c|c|c|c|c|c|c|c|c|c|c|c|c|c|c|}
\hline \multirow{2}{*}{ Cpd. } & \multirow{2}{*}{$\begin{array}{c}t_{R} \\
(\min )\end{array}$} & \multirow{2}{*}{$\begin{array}{l}\text { Elemental } \\
\text { Formula } \\
(\mathrm{M}+\mathrm{H})^{+}\end{array}$} & \multicolumn{2}{|c|}{ Mass $(m / z)$} & \multirow{2}{*}{$\begin{array}{l}\text { Error } \\
(\mathrm{ppm})\end{array}$} & \multirow{2}{*}{ DBE } & \multicolumn{2}{|c|}{ Fragmentation (MS/MS) } & \multicolumn{8}{|c|}{ Occurrence } \\
\hline & & & Exp. & Theo. & & & Mass $(m / z)$ & $\begin{array}{c}\text { Elemental } \\
\text { Formula }\end{array}$ & $\mathrm{DP}^{\mathrm{a}}$ & $\mathrm{T}^{\mathrm{b}}$ & $\mathrm{Fy}^{\mathrm{c}}$ & $\mathrm{Fr}^{\mathrm{d}}$ & $\mathrm{TFy}^{\mathrm{e}}$ & $\mathrm{TFr}^{\mathrm{f}}$ & $\mathrm{S}_{4}^{\mathrm{g}}$ & $\mathrm{S} 16^{\mathrm{h}}$ \\
\hline TP5 & 5.40 & $\mathrm{C}_{24} \mathrm{H}_{27} \mathrm{ClFN}_{4} \mathrm{O}_{3}$ & 473.1742 & 473.1750 & -1.69 & 13 & $\begin{array}{l}445.1759 \\
439.1637 \\
387.1273 \\
359.1309 \\
316.0954 \\
194.1297 \\
182.0912 \\
113.0709 \\
85.0768 \\
71.0616\end{array}$ & $\begin{array}{l}\mathrm{C}_{23} \mathrm{H}_{27} \mathrm{ClFN}_{4} \mathrm{O}_{2} \\
\mathrm{C}_{24} \mathrm{H}_{25} \mathrm{ClFN}_{4} \mathrm{O} \\
\mathrm{C}_{21} \mathrm{H}_{21} \mathrm{ClFN}_{2} \mathrm{O}_{2} \\
\mathrm{C}_{20} \mathrm{H}_{21} \mathrm{ClFN}_{2} \mathrm{O} \\
\mathrm{C}_{18} \mathrm{H}_{16} \mathrm{ClFNO} \\
\mathrm{C}_{10} \mathrm{H}_{16} \mathrm{~N}_{3} \mathrm{O} \\
\mathrm{C}_{8} \mathrm{H}_{12} \mathrm{~N}_{3} \mathrm{O}_{2} \\
\mathrm{C}_{5} \mathrm{H}_{9} \mathrm{~N}_{2} \mathrm{O} \\
\mathrm{C}_{4} \mathrm{H}_{9} \mathrm{~N}_{2} \\
\mathrm{C}_{3} \mathrm{H}_{7} \mathrm{~N}_{2}\end{array}$ & + & $+*$ & + & $+^{*}$ & $+^{*}$ & $+^{*}$ & - & $+^{*}$ \\
\hline TP6 & 6.89 & $\mathrm{C}_{24} \mathrm{H}_{27} \mathrm{ClFN}_{4} \mathrm{O}_{2}$ & 457.1805 & 457.1801 & 0.87 & 13 & $\begin{array}{c}439.1635 \\
355.1355 \\
194.1282 \\
168.1125 \\
129.0674 \\
113.0709 \\
111.0553 \\
84.0784 \\
\end{array}$ & $\begin{array}{l}\mathrm{C}_{24} \mathrm{H}_{25} \mathrm{ClFN}_{4} \mathrm{O} \\
\mathrm{C}_{21} \mathrm{H}_{21} \mathrm{ClFN}_{2} \\
\mathrm{C}_{10} \mathrm{H}_{16} \mathrm{~N}_{3} \mathrm{O} \\
\mathrm{C}_{8} \mathrm{H}_{14} \mathrm{~N}_{3} \mathrm{O} \\
\mathrm{C}_{5} \mathrm{H}_{9} \mathrm{~N}_{2} \mathrm{O}_{2} \\
\mathrm{C}_{5} \mathrm{H}_{9} \mathrm{~N}_{2} \mathrm{O} \\
\mathrm{C}_{5} \mathrm{H}_{7} \mathrm{~N}_{2} \mathrm{O} \\
\mathrm{C}_{5} \mathrm{H}_{10} \mathrm{~N}\end{array}$ & + & + & + & + & + & + & + & + \\
\hline TP7 & 2.42 & $\mathrm{C}_{18} \mathrm{H}_{23} \mathrm{~N}_{4} \mathrm{O}_{3}$ & 343.1778 & 343.1765 & 4.08 & 10 & \begin{tabular}{c|}
257.131 \\
245.1286 \\
214.0869 \\
201.0787 \\
194.1282 \\
168.1123 \\
142.0981 \\
113.0705 \\
\end{tabular} & $\begin{array}{l}\mathrm{C}_{15} \mathrm{H}_{17} \mathrm{~N}_{2} \mathrm{O}_{2} \\
\mathrm{C}_{14} \mathrm{H}_{17} \mathrm{~N}_{2} \mathrm{O}_{2} \\
\mathrm{C}_{13} \mathrm{H}_{12} \mathrm{NO}_{2} \\
\mathrm{C}_{12} \mathrm{H}_{11} \mathrm{NO}_{2} \\
\mathrm{C}_{10} \mathrm{H}_{16} \mathrm{~N}_{3} \mathrm{O} \\
\mathrm{C}_{8} \mathrm{H}_{14} \mathrm{~N}_{3} \mathrm{O} \\
\mathrm{C}_{6} \mathrm{H}_{12} \mathrm{~N}_{3} \mathrm{O} \\
\mathrm{C}_{5} \mathrm{H}_{9} \mathrm{~N}_{2} \mathrm{O}\end{array}$ & + & - & $+^{*}$ & $+^{*}$ & $+^{*}$ & $+^{*}$ & $+^{*}$ & + \\
\hline TP8 & 5.93 & $\mathrm{C}_{24} \mathrm{H}_{27} \mathrm{ClFN}_{4} \mathrm{O}_{2}$ & 457.1810 & 457.1801 & 1.97 & 13 & $\begin{array}{c}371.1311 \\
345.1139 \\
328.0858 \\
194.1247 \\
113.0713 \\
70.0657\end{array}$ & $\begin{array}{c}\mathrm{C}_{21} \mathrm{H}_{21} \mathrm{ClFN}_{2} \mathrm{O} \\
\mathrm{C}_{19} \mathrm{H}_{19} \mathrm{ClFN}_{2} \mathrm{O} \\
\mathrm{C}_{19} \mathrm{H}_{16} \mathrm{ClFNO} \\
\mathrm{C}_{10} \mathrm{H}_{16} \mathrm{~N}_{3} \mathrm{O} \\
\mathrm{C}_{5} \mathrm{H}_{9} \mathrm{~N}_{2} \mathrm{O} \\
\mathrm{C}_{4} \mathrm{H}_{8} \mathrm{~N}\end{array}$ & $+^{*}$ & + & $+^{*}$ & + & + & + & + & $+*$ \\
\hline
\end{tabular}


Table 3. Cont

\begin{tabular}{|c|c|c|c|c|c|c|c|c|c|c|c|c|c|c|c|c|}
\hline \multirow{2}{*}{ Cpd. } & \multirow{2}{*}{$\begin{array}{c}t_{R} \\
(\mathrm{~min})\end{array}$} & \multirow{2}{*}{$\begin{array}{l}\text { Elemental } \\
\text { Formula } \\
(\mathrm{M}+\mathrm{H})^{+}\end{array}$} & \multicolumn{2}{|c|}{ Mass $(m / z)$} & \multirow{2}{*}{$\begin{array}{l}\text { Error } \\
(\mathrm{ppm})\end{array}$} & \multirow{2}{*}{ DBE } & \multicolumn{2}{|c|}{ Fragmentation (MS/MS) } & \multicolumn{8}{|c|}{ Occurrence } \\
\hline & & & Exp. & Theo. & & & Mass $(m / z)$ & $\begin{array}{c}\text { Elemental } \\
\text { Formula }\end{array}$ & $\mathrm{DP}^{\mathrm{a}}$ & $\mathrm{T}^{\mathrm{b}}$ & $\mathrm{Fy}^{\mathrm{c}}$ & $\mathrm{Fr}^{\mathrm{d}}$ & TFy ${ }^{\mathbf{e}}$ & $\mathrm{TFr}^{\mathrm{f}}$ & $\mathrm{S} 4^{\mathrm{g}}$ & $\mathrm{S} 16^{\mathrm{h}}$ \\
\hline TP9 & 5.28 & $\mathrm{C}_{24} \mathrm{H}_{27} \mathrm{ClFN}_{4} \mathrm{O}_{4}$ & 489.1704 & 489.1699 & 1.02 & 13 & $\begin{array}{c}471.1599 \\
443.1607 \\
387.1255 \\
361.1126 \\
210.1219 \\
129.0662 \\
113.0707 \\
111.0547 \\
69.0505 \\
\end{array}$ & $\begin{array}{l}\mathrm{C}_{24} \mathrm{H}_{25} \mathrm{ClFN}_{4} \mathrm{O}_{3} \\
\mathrm{C}_{23} \mathrm{H}_{25} \mathrm{ClFN}_{4} \mathrm{O}_{2} \\
\mathrm{C}_{21} \mathrm{H}_{21} \mathrm{ClFN}_{4} \mathrm{O}_{2} \\
\mathrm{C}_{19} \mathrm{H}_{19} \mathrm{ClFN}_{2} \mathrm{O}_{2} \\
\mathrm{C}_{10} \mathrm{H}_{16} \mathrm{~N}_{3} \mathrm{O}_{2} \\
\mathrm{C}_{5} \mathrm{H}_{9} \mathrm{~N}_{2} \mathrm{O}_{2} \\
\mathrm{C}_{5} \mathrm{H}_{9} \mathrm{~N}_{2} \mathrm{O} \\
\mathrm{C}_{5} \mathrm{H}_{7} \mathrm{~N}_{2} \mathrm{O} \\
\mathrm{C}_{3} \mathrm{H}_{5} \mathrm{~N}_{2}\end{array}$ & $+*$ & + & - & $+^{*}$ & + & + & - & - \\
\hline TP10 & 5.09 & $\mathrm{C}_{24} \mathrm{H}_{27} \mathrm{ClFN}_{4} \mathrm{O}_{3}$ & 473.1735 & 473.1750 & -3.17 & 13 & \begin{tabular}{l|}
455.1604 \\
387.1265 \\
326.0723 \\
300.0601 \\
210.1259 \\
194.1274 \\
142.0974 \\
113.0708 \\
\end{tabular} & $\begin{array}{l}\mathrm{C}_{23} \mathrm{H}_{27} \mathrm{ClFN}_{4} \mathrm{O}_{2} \\
\mathrm{C}_{21} \mathrm{H}_{21} \mathrm{ClFN}_{2} \mathrm{O}_{2} \\
\mathrm{C}_{19} \mathrm{H}_{14} \mathrm{ClFNO} \\
\mathrm{C}_{17} \mathrm{H}_{12} \mathrm{ClFNO} \\
\mathrm{C}_{10} \mathrm{H}_{16} \mathrm{~N}_{3} \mathrm{O}_{2} \\
\mathrm{C}_{10} \mathrm{H}_{16} \mathrm{~N}_{3} \mathrm{O} \\
\mathrm{C}_{6} \mathrm{H}_{12} \mathrm{~N}_{3} \mathrm{O} \\
\mathrm{C}_{5} \mathrm{H}_{9} \mathrm{~N}_{2} \mathrm{O}\end{array}$ & $+*$ & + & $+*$ & $+^{*}$ & + & + & $+^{*}$ & $+^{*}$ \\
\hline TP11 & 6.03 & $\mathrm{C}_{24} \mathrm{H}_{28} \mathrm{ClN}_{4} \mathrm{O}_{2}$ & 439.1891 & 439.1895 & -0.91 & 13 & $\begin{array}{l}353.1431 \\
196.1421 \\
113.0712\end{array}$ & $\begin{array}{l}\mathrm{C}_{21} \mathrm{H}_{22} \mathrm{ClN}_{2} \mathrm{O} \\
\mathrm{C}_{10} \mathrm{H}_{18} \mathrm{~N}_{3} \mathrm{O} \\
\mathrm{C}_{5} \mathrm{H}_{9} \mathrm{~N}_{2} \mathrm{O}\end{array}$ & $+^{*}$ & $+^{*}$ & $+{ }^{*}$ & $+^{*}$ & $+^{*}$ & $+^{*}$ & $+^{*}$ & $+^{*}$ \\
\hline TP12 & 4.54 & $\mathrm{C}_{24} \mathrm{H}_{28} \mathrm{FN}_{4} \mathrm{O}_{3}$ & 439.2139 & 439.2140 & -0.23 & 13 & $\begin{array}{l}353.1632 \\
327.1488 \\
310.1222 \\
194.1247 \\
113.0707\end{array}$ & $\begin{array}{l}\mathrm{C}_{21} \mathrm{H}_{22} \mathrm{FN}_{2} \mathrm{O}_{2} \\
\mathrm{C}_{19} \mathrm{H}_{20} \mathrm{FN}_{2} \mathrm{O}_{2} \\
\mathrm{C}_{19} \mathrm{H}_{17} \mathrm{FNO}_{2} \\
\mathrm{C}_{10} \mathrm{H}_{16} \mathrm{~N}_{3} \mathrm{O} \\
\mathrm{C}_{5} \mathrm{H}_{9} \mathrm{~N}_{2} \mathrm{O}\end{array}$ & $+^{*}$ & $+^{*}$ & $+*$ & $+*$ & + & + & + & $+*$ \\
\hline TP13 & 3.89 & $\mathrm{C}_{24} \mathrm{H}_{27} \mathrm{~N}_{4} \mathrm{O}_{4}$ & 435.2036 & 435.2027 & 2.07 & 14 & $\begin{array}{l}194.1297 \\
168.1122 \\
113.0705 \\
\end{array}$ & $\begin{array}{l}\mathrm{C}_{10} \mathrm{H}_{16} \mathrm{~N}_{3} \mathrm{O} \\
\mathrm{C}_{8} \mathrm{H}_{14} \mathrm{~N}_{3} \mathrm{O} \\
\mathrm{C}_{5} \mathrm{H}_{9} \mathrm{~N}_{2} \mathrm{O} \\
\end{array}$ & $+^{*}$ & - & $+*$ & $+^{*}$ & $+^{*}$ & $+^{*}$ & $+*$ & $+^{*}$ \\
\hline TP14 & 6.35 & $\mathrm{C}_{24} \mathrm{H}_{27} \mathrm{ClFN}_{4} \mathrm{O}_{2}$ & 457.1802 & 457.1801 & 0.22 & 13 & $\begin{array}{l}142.0964 \\
113.0709 \\
\end{array}$ & $\begin{array}{l}\mathrm{C}_{6} \mathrm{H}_{12} \mathrm{~N}_{3} \mathrm{O} \\
\mathrm{C}_{5} \mathrm{H}_{9} \mathrm{~N}_{2} \mathrm{O}\end{array}$ & - & $+^{*}$ & $+{ }^{*}$ & $+^{*}$ & $+*$ & $+^{*}$ & $+*$ & $+^{*}$ \\
\hline TP15 & 6.57 & $\mathrm{C}_{24} \mathrm{H}_{23} \mathrm{ClFN}_{4} \mathrm{O}$ & 437.1516 & 437.1539 & -5.26 & 15 & $\begin{array}{l}325.0877 \\
308.0628 \\
113.0710\end{array}$ & $\begin{array}{c}\mathrm{C}_{19} \mathrm{H}_{15} \mathrm{ClFN}_{2} \\
\mathrm{C}_{19} \mathrm{H}_{12} \mathrm{ClFN} \\
\mathrm{C}_{5} \mathrm{H}_{9} \mathrm{~N}_{2} \mathrm{O}\end{array}$ & $+^{*}$ & $+^{*}$ & $+^{*}$ & $+^{*}$ & $+^{*}$ & $+^{*}$ & $+^{*}$ & $+^{*}$ \\
\hline
\end{tabular}


Table 3. Cont

\begin{tabular}{|c|c|c|c|c|c|c|c|c|c|c|c|c|c|c|c|c|}
\hline \multirow{2}{*}{ Cpd. } & \multirow{2}{*}{$\begin{array}{c}t_{R} \\
(\min )\end{array}$} & \multirow{2}{*}{$\begin{array}{l}\text { Elemental } \\
\text { Formula } \\
(\mathrm{M}+\mathrm{H})^{+}\end{array}$} & \multicolumn{2}{|c|}{ Mass $(m / z)$} & \multirow{2}{*}{$\begin{array}{l}\text { Error } \\
(\mathrm{ppm})\end{array}$} & \multirow{2}{*}{ DBE } & \multicolumn{2}{|c|}{ Fragmentation (MS/MS) } & \multicolumn{8}{|c|}{ Occurrence } \\
\hline & & & Exp. & Theo. & & & Mass $(m / z)$ & $\begin{array}{c}\text { Elemental } \\
\text { Formula }\end{array}$ & $D^{a}{ }^{a}$ & $\mathrm{~T}^{\mathrm{b}}$ & $\mathrm{Fy}^{\mathrm{c}}$ & $\mathrm{Fr}^{\mathrm{d}}$ & $\mathrm{TFy}^{\mathrm{e}}$ & $\mathrm{TFr}^{\mathrm{f}}$ & $\mathrm{S} 4^{\mathrm{g}}$ & $\mathrm{S} 16^{\mathrm{h}}$ \\
\hline TP16 & 6.35 & $\mathrm{C}_{24} \mathrm{H}_{23} \mathrm{ClFN}_{4} \mathrm{O}_{2}$ & 453.1474 & 453.1488 & -3.09 & 15 & $\begin{array}{l}435.1362 \\
351.1076 \\
325.0861 \\
308.0643 \\
129.0654 \\
113.0707 \\
111.0547\end{array}$ & $\begin{array}{l}\mathrm{C}_{24} \mathrm{H}_{21} \mathrm{ClFN}_{4} \mathrm{O} \\
\mathrm{C}_{42} \mathrm{H}_{17} \mathrm{ClFN}_{2} \\
\mathrm{C}_{1} \mathrm{H}_{15} \mathrm{ClFN}_{2} \\
\mathrm{C}_{19} \mathrm{H}_{12} \mathrm{ClFN} \mathrm{ClFN}_{5} \mathrm{H}_{9} \mathrm{~N}_{2} \mathrm{O}_{2} \\
\mathrm{C}_{5} \mathrm{H}_{9} \mathrm{~N}_{2} \mathrm{O} \\
\mathrm{C}_{5} \mathrm{H}_{7} \mathrm{~N}_{2} \mathrm{O}\end{array}$ & - & $+^{*}$ & - & - & $+^{*}$ & $+{ }^{*}$ & $+^{*}$ & - \\
\hline TP17 & 6.66 & $\mathrm{C}_{24} \mathrm{H}_{21} \mathrm{ClFN}_{4} \mathrm{O}_{2}$ & 451.1304 & 451.1332 & -6.21 & 16 & $\begin{array}{c}325.0805 \\
308.0537 \\
258.0515 \\
127.0502 \\
99.0568 \\
\end{array}$ & $\begin{array}{l}\mathrm{C}_{19} \mathrm{H}_{15} \mathrm{ClFN}_{2} \\
\mathrm{C}_{19} \mathrm{H}_{12} \mathrm{ClFN} \\
\mathrm{C}_{15} \mathrm{H}_{10} \mathrm{ClFN} \\
\mathrm{C}_{5} \mathrm{H}_{7} \mathrm{~N}_{2} \mathrm{O}_{2} \\
\mathrm{C}_{4} \mathrm{H}_{7} \mathrm{~N}_{2} \mathrm{O}\end{array}$ & $+^{*}$ & $+^{*}$ & - & - & $+^{*}$ & $+{ }^{*}$ & $+^{*}$ & - \\
\hline TP18 & 6.52 & $\mathrm{C}_{24} \mathrm{H}_{28} \mathrm{FN}_{4} \mathrm{O}$ & 407.2229 & 407.2242 & -3.19 & 13 & $\begin{array}{l}321.1769 \\
196.1423 \\
113.0710\end{array}$ & $\begin{array}{c}\mathrm{C}_{21} \mathrm{H}_{22} \mathrm{FN}_{2} \\
\mathrm{C}_{10} \mathrm{H}_{18} \mathrm{~N}_{3} \mathrm{O} \\
\mathrm{C}_{5} \mathrm{H}_{9} \mathrm{~N}_{2} \mathrm{O}\end{array}$ & + & + & + & + & + & + & + & + \\
\hline
\end{tabular}

${ }^{*}$ Detected in trace level. ${ }^{\mathrm{a}}$ Direct photolysis; ${ }^{\mathrm{b}} \mathrm{TiO}_{2} ;{ }^{\mathrm{c}} \mathrm{FeOOH}$ (yellow); ${ }^{\mathrm{d}} \mathrm{Fe}_{2} \mathrm{O}_{3}$ (red); ${ }^{\mathrm{e}} \mathrm{TiO}_{2}-\mathrm{Fe}_{2} \mathrm{O}_{3}$ yellow; ${ }^{\mathrm{f}} \mathrm{TiO}_{2}-\mathrm{Fe}_{2} \mathrm{O}_{3}$ red; ${ }^{\mathrm{g}}$ Serdolect $4 \mathrm{mg} ;{ }^{\mathrm{h}} \mathrm{Serdolect} 16 \mathrm{mg}$. 


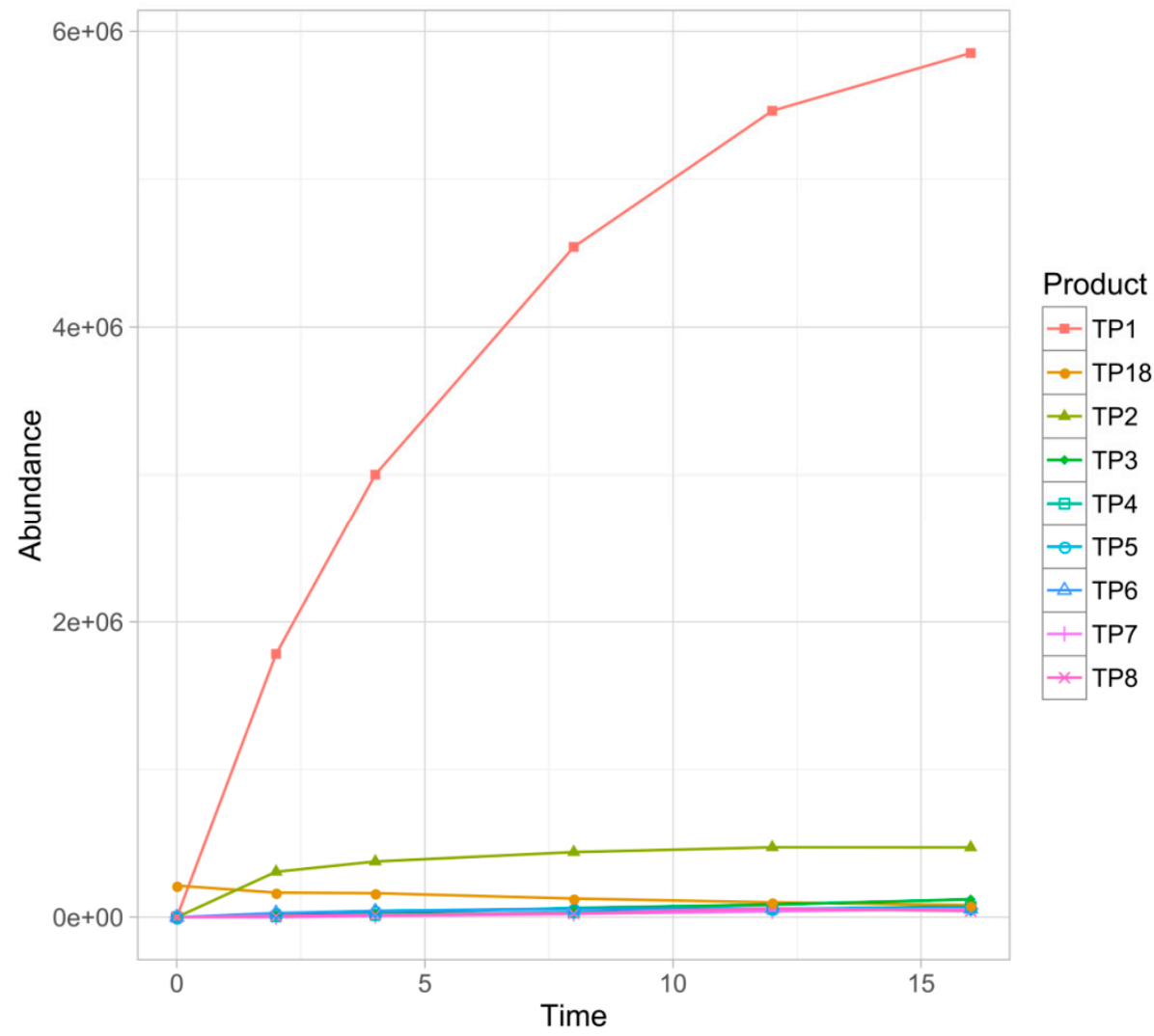

Figure 2. Evolution profiles of the phototransformation products (TPs) formed in the direct photolysis.

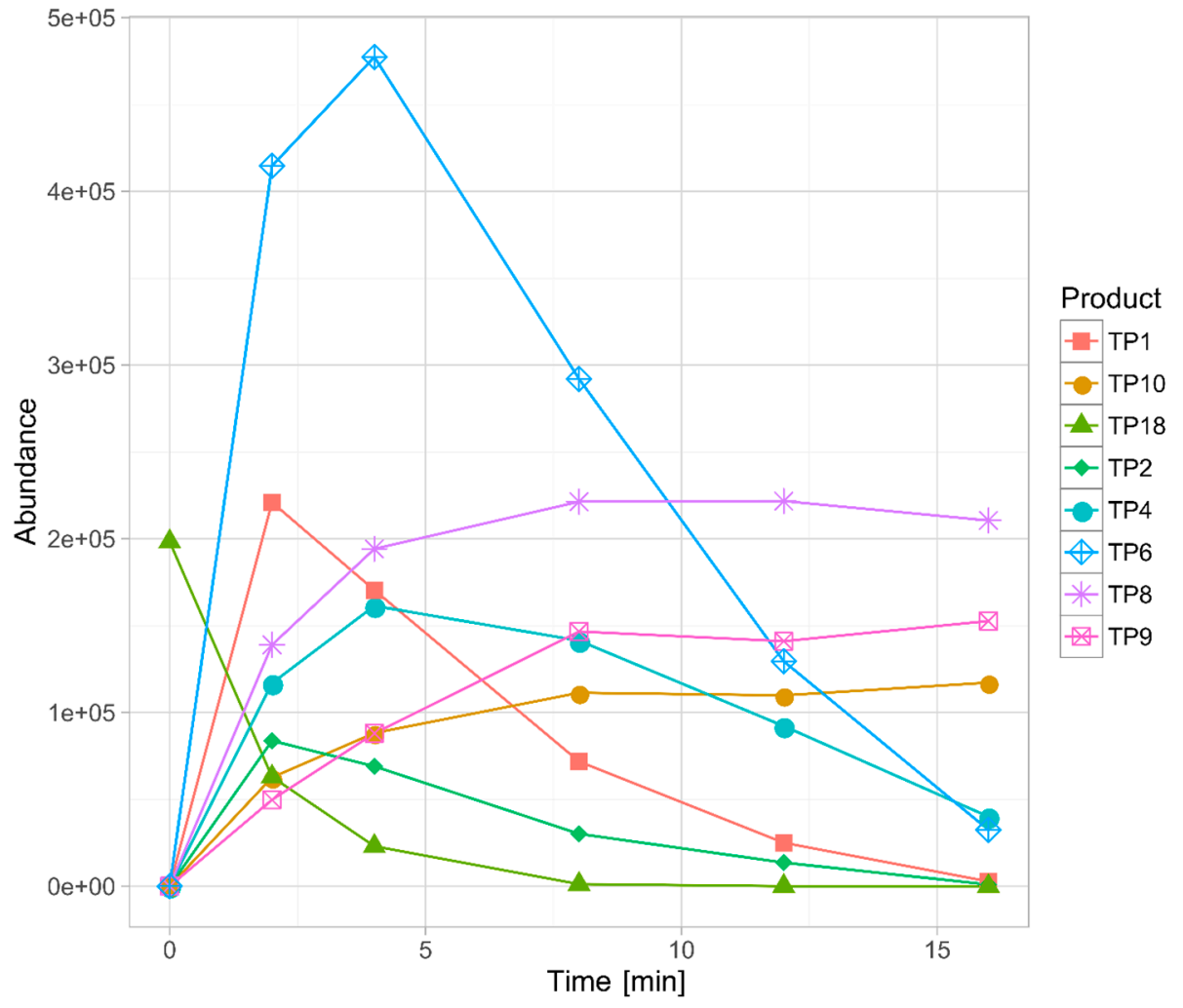

Figure 3. Evolution profiles of the TPs formed in the $\mathrm{TiO}_{2}$ photocatalysis. 

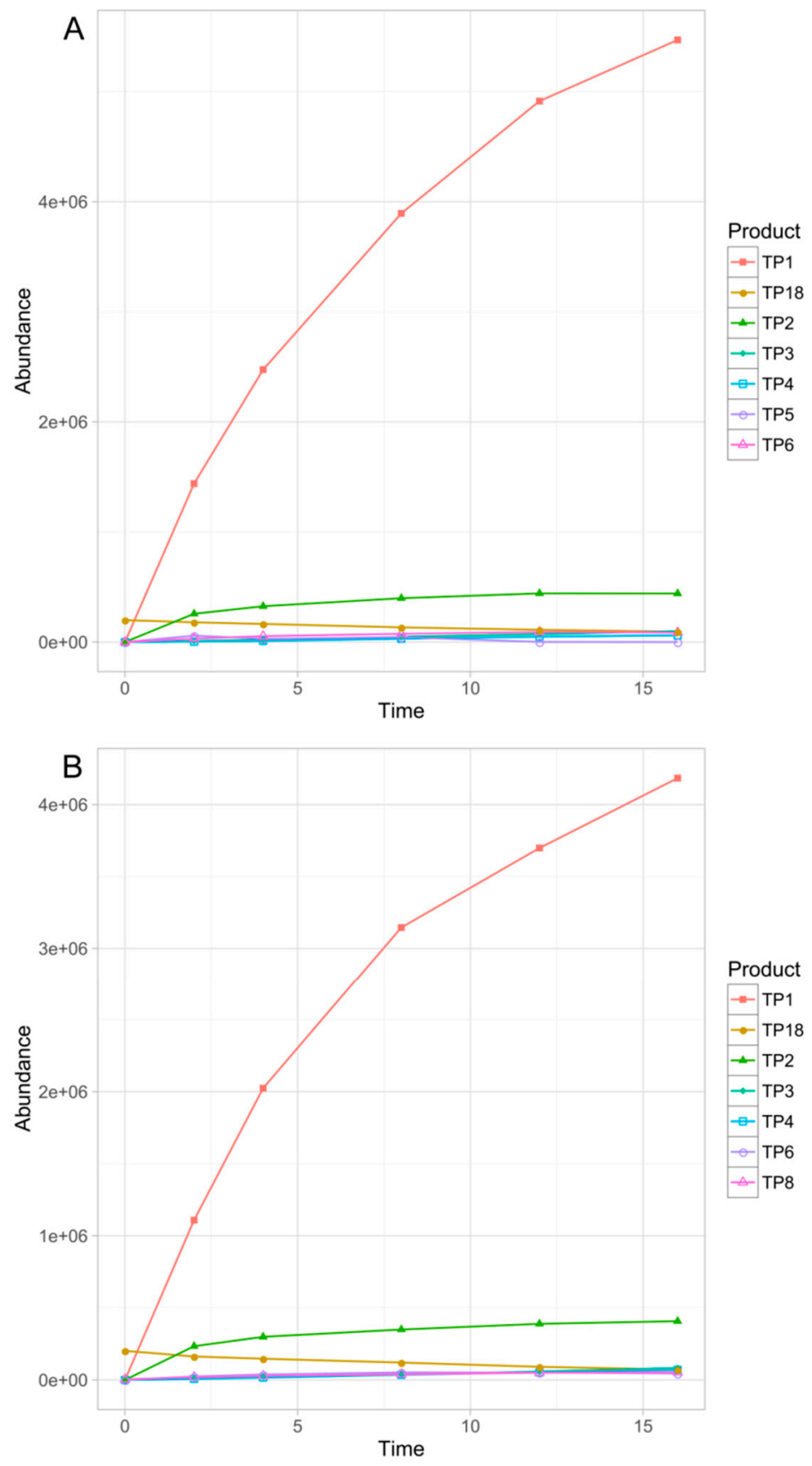

Figure 4. Evolution profiles of the TPs formed in $\mathrm{FeOOH}(\mathbf{A})$ and $\mathrm{Fe}_{2} \mathrm{O}_{3}$ (B) photocatalysis. 

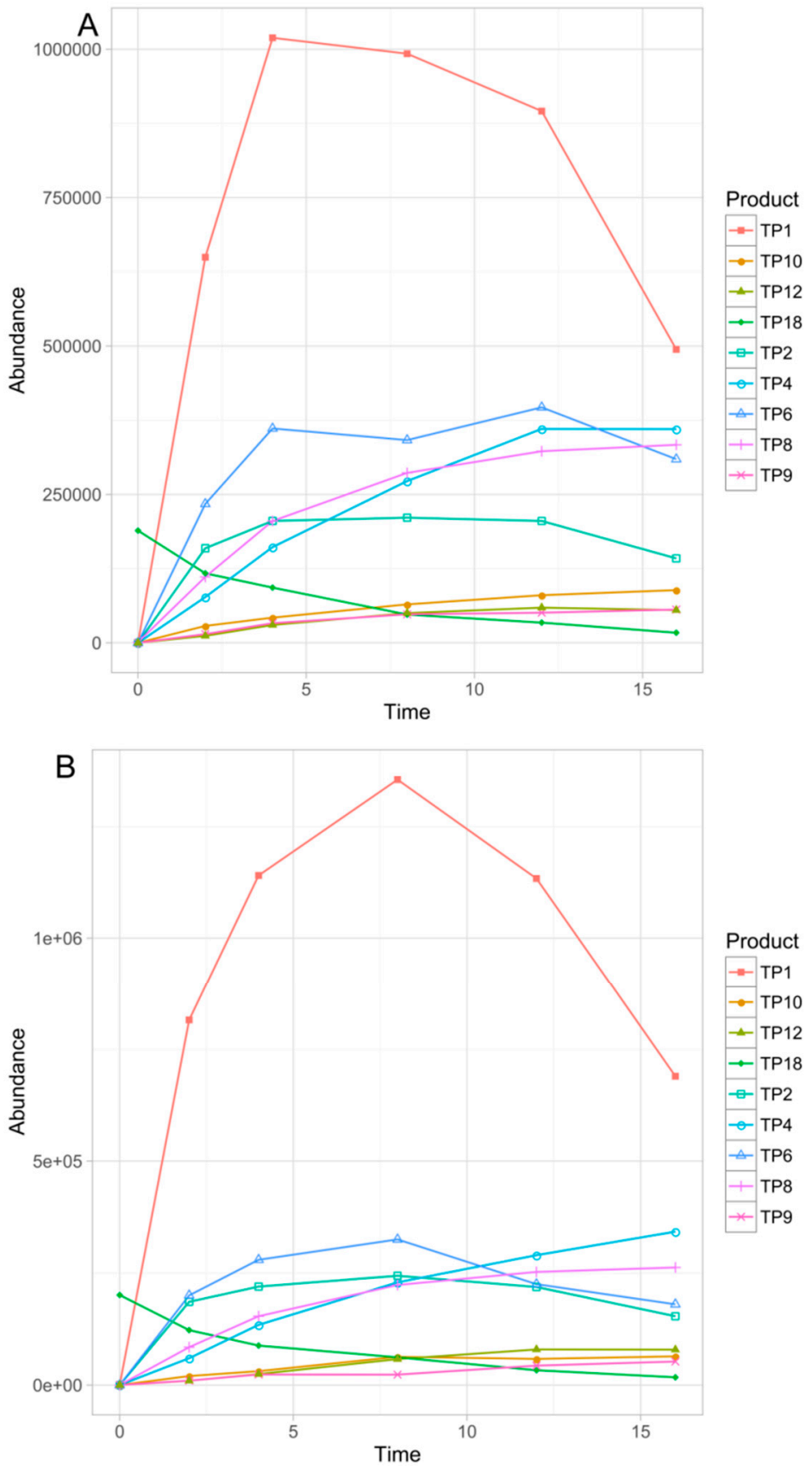

Figure 5. Evolution profiles of the TPs formed in $\mathrm{TiO}_{2}-\mathrm{FeOOH}(\mathbf{A})$ and $\mathrm{TiO}_{2}-\mathrm{Fe}_{2} \mathrm{O}_{3}(\mathbf{B})$ photocatalysis. 

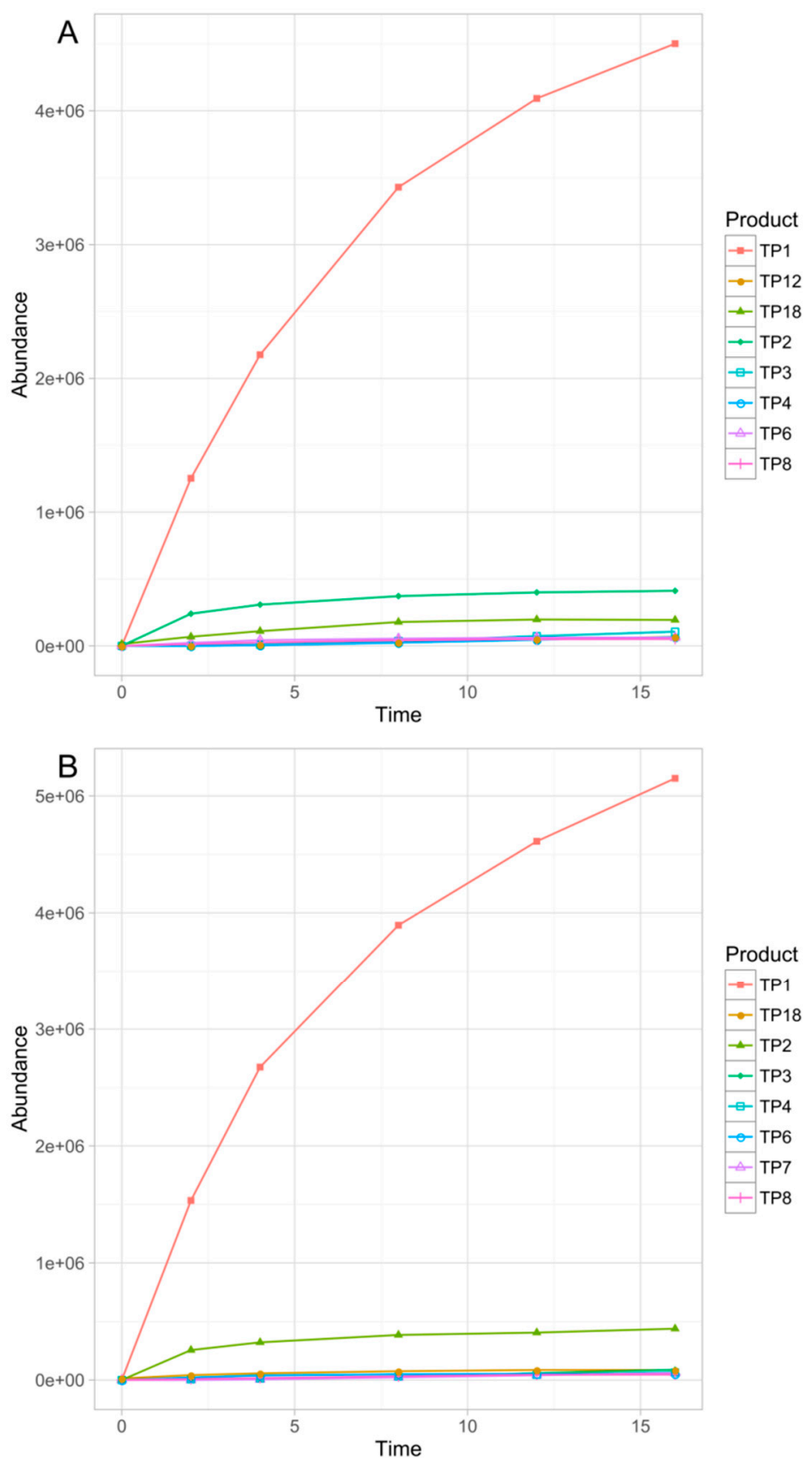

Figure 6. Evolution profiles of the TPs formed in Serdolect ${ }^{\circledR} 4 \mathrm{mg}$ (A) and Serdolect ${ }^{\circledR} 16 \mathrm{mg}$ (B) experiments.

MS/MS spectrum of sertindole (CID energy $=25.1 \mathrm{eV}$ ) is shown in Figure S1. The fragmentation began with the elimination of an imidazolidine-2-one and the formation of $\mathrm{m} / \mathrm{z} 355.1379$ ion, then the elimination of an ethyl fragment $(m / z$ 329.1200). Next the gradual decomposition of a piperidine ring took place $(\mathrm{m} / \mathrm{z} 298.0765$ and $\mathrm{m} / \mathrm{z} 270.0464$ ions). The main fragmentation ion- $m / z$ 113.0713-represented 1-ethenyl-imidazolidine-2-one, fragment common for the majority of TPs with an unchanged imidazolidine-2-one ring. The ions representing various fragments of 1-[2-(piperidine-1-yl)ethyl]imidazolidine-2-one were also present in the spectrum $(\mathrm{m} / \mathrm{z} 196.1443, \mathrm{~m} / \mathrm{z}$ 168.1131, and $m / z 142.0967$ ions).

$\mathrm{TP} 1$ was the major product in almost all of the experiments. Only in the case of $\mathrm{TiO}_{2}$-mediated photocatalysis its abundance did not significantly diverge from the other TPs. In the direct photolysis (its DAD peak area equaled over $290 \%$ of sertindole peak area after 16 min of irradiation), $\mathrm{Fe}_{2} \mathrm{O}_{3}$ and 
$\mathrm{FeOOH}$ photocatalysis as well as in the S4 and S16 experiments, the abundance of TP1 was over one order of magnitude higher than any of the remaining TPs. Its concentration was increasing up to $16 \mathrm{~min}$ when the experiments were terminated. In the mixed-catalysts experiments, TP1 was also the main product, however, its abundance was less than one order of magnitude higher than the other TPs. Its kinetic behavior was also different. In the $\mathrm{TiO}_{2}-\mathrm{FeOOH}$ experiment $\mathrm{TP} 1$ concentration was increasing up to $4 \mathrm{~min}$ and then the product started to decompose. In the $\mathrm{TiO}_{2}-\mathrm{Fe}_{2} \mathrm{O}_{3}$ the photocatalysis decomposition of TP1 started after $8 \mathrm{~min}$. In the $\mathrm{TiO}_{2}$-mediated photocatalysis TP1 was the second most abundant product and its kinetic behavior was similar to that observed in $\mathrm{TiO}_{2}-\mathrm{FeOOH}$ experiment. TP1 contained one additional oxygen atom instead of chlorine. Based on its MS/MS spectrum (Figure S2), the whole 1-[2-(piperidine-1-yl)ethyl]imidazolidine-2-one fragment remained unchanged. Therefore, the additional oxygen was attached to the phenylindole fragment. Taking into account that in the case of this product dechlorination took place, TP1 was probably a product of chlorine substitution with a hydroxyl group.

TP2 was the second most abundant product in all the experiments excluding $\mathrm{TiO}_{2}, \mathrm{TiO}_{2}-\mathrm{FeOOH}$, and $\mathrm{TiO}_{2}-\mathrm{Fe}_{2} \mathrm{O}_{3}$ photocatalysis, and was probably a product of double N-oxidation of TP1. This assumption was based on a very feasible loss of two additional oxygen atoms $(\mathrm{m} / z$ 439.2115 and $m / z$ 421.1996) without any fragmentation of 1-[2-(piperidine-1-yl)ethyl]imidazolidine-2-one fragment (Figure S3).

TP3 was not detected in the $\mathrm{TiO}_{2}$-catalyzed sample, and in $\mathrm{TiO}_{2}-\mathrm{FeOOH}$ as well as in $\mathrm{TiO}_{2}-\mathrm{Fe}_{2} \mathrm{O}_{3}$ experiments only its traces were detected. In the remaining experiments it was the third most abundant product (except the S4 where TP18 was more abundant). TP3 was probably a product of TP1 defluorination-its fragmentation pattern (Figure S4) was very similar to TP1 but the fragmentation ions did not contain fluorine.

TP4 was formed in all the experiments and its highest abundance was found in the $\mathrm{TiO}_{2}-\mathrm{FeOOH}$ and $\mathrm{TiO}_{2}-\mathrm{Fe}_{2} \mathrm{O}_{3}$ samples. It was a product of $\mathrm{TP} 1$ hydroxylation and dehydrogenation. An additional oxygen was probably attached to a phenylindole fragment based on the presence of 1-[2-(piperidine-1-yl)ethyl]imidazolidine-2-one fragment without any oxygen atom (Figure S5). However, an unusual fragmentation of this ion $(\mathrm{m} / \mathrm{z} 168.1123$ and $m / z 142.0950)$ suggested that dehydrogenation took place in the piperidine ring.

TP5 was found in trace level in the majority of experiments and was completely absent in S16 sample. However, it was the sixth most abundant product in the direct photolysis and FeOOH-catalyzed samples. TP5 was a product of addition of two oxygen atoms to a sertindole molecule. Both of them were eliminated quite easily $(\mathrm{m} / \mathrm{z} 439.1637$, Figure S6), therefore, they were rather not attached to an aromatic rings. Nevertheless, a significant difference in the behavior of these two oxygen atoms was observed-only one of them was present in the ions containing the residuals of a piperidine ring (such as $\mathrm{m} / \mathrm{z} 315.0954$ and $\mathrm{m} / \mathrm{z}$ 182.0912). Such observations and the presence of an unchanged 1-ethenyl-imidazolidine-2-one fragment ( $\mathrm{m} / \mathrm{z} 113.0709 \mathrm{ion})$ suggested that TP5 was a piperidine $\mathrm{N}$-oxide containing a hydroxyl group attached to a piperidine ring. A very similar fragmentation pattern was observed in the case of TP10 (Figure S11) which was probably an isomer of TP5.

TP6 was detected in all the experiments and was the major product in the $\mathrm{TiO}_{2}, \mathrm{TiO}_{2}-\mathrm{FeOOH}$, and $\mathrm{TiO}_{2}-\mathrm{Fe}_{2} \mathrm{O}_{3}$ samples. In this case the hydroxylation of an imidazolidine-2-one ring took place, which was confirmed by the presence of $m / z 129.0674$ (1-ethenyl-imidazolidine-2-one with an additional oxygen) and $\mathrm{m} / \mathrm{z} 111.0553$ (dehydrogenated 1-ethenyl-imidazolidine-2-one) ions (Figure S7). The presence of $\mathrm{m} / \mathrm{z} 355.1355$ ion confirmed that hydroxylation did not take place in an ethyl fragment.

TP7, the product of elimination of 4-fluorophenyl from TP4, generally was detected in trace level. In the $\mathrm{TiO}_{2}$-catalyzed experiment it was completely absent. Only in cases of the direct photolysis and S16 relatively high abundances were observed. The fragmentation pattern of TP7 (Figure S8) was in general very similar to TP4 but the fragmentation ions did not contain the 4-fluorophenyl fragment. Both additional oxygen atoms were not feasibly eliminated (which suggested aromatic hydroxylation) and a piperidine ring was susceptible to the fragmentation $(\mathrm{m} / \mathrm{z} 168.1123$ and $\mathrm{m} / \mathrm{z} 142.0981$ ions). 
TP8 was formed during all the conducted experiments, however, in the direct photolysis, $\mathrm{FeOOH}$ photocatalysis and S16 experiments it was detected in trace level. The product was formed probably as a consequence of aromatic hydroxylation of sertindole. The ions representing 1-[2-(piperidine-1-yl)ethyl]imidazolidine-2-one and its fragments remained unchanged $(\mathrm{m} / \mathrm{z} 142.1247$ and $m / z 113.0713$ ions, Figure S9).

TP9, detected in the $\mathrm{TiO}_{2}, \mathrm{TiO}_{2}-\mathrm{FeOOH}, \mathrm{TiO}_{2}-\mathrm{Fe}_{2} \mathrm{O}_{3}$ samples and as the traces in the direct photolysis as well as in $\mathrm{Fe}_{2} \mathrm{O}_{3}$ photocatalysis, possessed three additional oxygen atoms. One of them was attached to 1-ethenyl-imidazolidine-2-one fragment $(\mathrm{m} / \mathrm{z} 129.0662$ and $\mathrm{m} / \mathrm{z} 111.0547$ ions, Figure S10), the second one to a piperidine ring ( $\mathrm{m} / \mathrm{z} 210.1219 \mathrm{ion})$, and the third one was probably an aromatic hydroxyl group.

TP11 contained one additional hydroxyl group instead of chlorine. The fragmentation patterns of TP11 and the parent compound were very similar (Figure S12), therefore, in the case of this product the substitution of fluorine with a hydroxyl group probably took place. TP11 was found in all the experiments, however, only at a trace level.

TP12 was formed probably as a consequence of aromatic hydroxylation of TP1. In its MS/MS spectrum (Figure S13) unmodified 1-[2-(piperidine-1-yl)ethyl]imidazolidine-2-one fragments were observed $(\mathrm{m} / \mathrm{z} 194.1247$ and $\mathrm{m} / \mathrm{z} 113.0707$ ions) and the additional hydroxyl groups were not eliminated easily (e.g., $m / z 310.1222$ and $m / z 327.1488$ ions). TP12 was generally detected as the traces and only in the $\mathrm{TiO}_{2}-\mathrm{Fe}_{2} \mathrm{O}_{3}, \mathrm{TiO}_{2}-\mathrm{FeOOH}$, and $\mathrm{S} 4$ experiments it was present in a relatively high level.

TP13 possessed an additional double bond in a piperidine ring (unusually high abundance of $\mathrm{m} / \mathrm{z}$ 168.1122 ion-a consequence of piperidine fragmentation, Figure S14) and three additional oxygen atoms. None of them was feasibly eliminated, therefore, all of them probably formed aromatic hydroxyl groups. TP13 was generally a low-abundant product (in the $\mathrm{TiO}_{2}$ photocatalysis the product was not detected).

TP14 was a product of sertindole piperidine ring hydroxylation which was confirmed by an unusually high abundance of 1-[2-(piperidine-1-yl)ethyl]imidazolidine-2-one fragmentation ion $(\mathrm{m} / \mathrm{z}$ 142.0964) and an unchanged 1-ethenyl-imidazolidine-2-one fragment $(\mathrm{m} / \mathrm{z}$ 113.0709). TP14 was not formed during the direct photolysis. In the remaining experiments it was detected at a trace level.

TP15 possessed two additional double bonds which were probably located in a piperidine ring $(\mathrm{m} / \mathrm{z} 325.0877$ and $\mathrm{m} / \mathrm{z} 308.0628$ ions), and was a low-abundant product in all the experiments.

TP16 was a product of TP15 hydroxylation. The location of two additional double bonds was confirmed, similarly as in the case of TP15, by the presence of $\mathrm{m} / \mathrm{z} 325.0861, \mathrm{~m} / \mathrm{z} 308.0643$, and additionally $\mathrm{m} / \mathrm{z} 351.1076$ (Figure S17). A hydroxyl group was attached to an imidazolidine-2-one ring, which was confirmed by the presence of $\mathrm{m} / \mathrm{z} 129.0654$ and $\mathrm{m} / \mathrm{z} 111.0547$ ions. TP16 was not detected in the direct photolysis, $\mathrm{TiO}_{2}-\mathrm{Fe}_{2} \mathrm{O}_{3}, \mathrm{TiO}_{2}-\mathrm{FeOOH}$, and $\mathrm{S} 16$ experiments. In the remaining experiments it was detected at a trace level.

The dehydrogenation of TP16 gave TP17 product whose occurrence was very similar. Its fragmentation pattern is shown in Figure S18.

TP18, a dechlorinated sertindole derivative (Figure S19), was a particularly interesting product. In all the experiments, excluding S4 and S16, this species was present from the beginning of the experiments and its concentration was decreasing up to $16 \mathrm{~min}$. Unsurprisingly, the fastest degradation was observed in the $\mathrm{TiO}_{2}$ photocatalysis. These observations suggested that TP18 is an impurity, present in the sertindole standard but absent in both pharmaceutical formulations. Noteworthy is that in the S4 and S16 experiments the opposite kinetic behavior of this product was observed-it was almost undetectable at the beginning, and its concentration was increasing up to 16 min. Moreover, TP18 reached a significantly higher concentration in S4 than S16 experiment.

Proposed photodegradation pathway of sertindole is presented in Figure 7. 


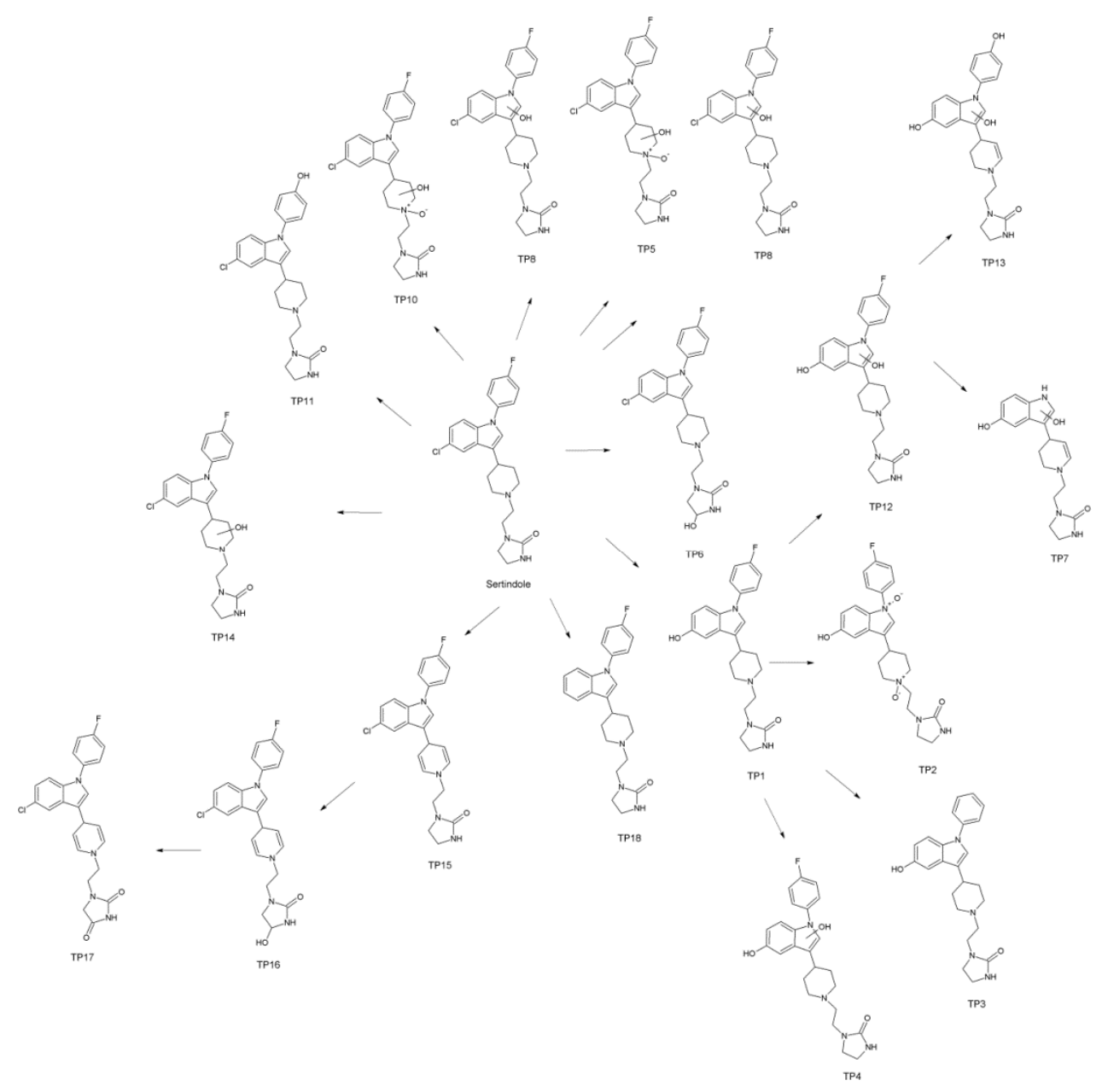

Figure 7. Proposed pathway of sertindole photodegradation.

\subsection{Chemometric Data Analysis}

In order to perform the multivariate chemometric analysis, all the obtained LC-MS profiles (48 chromatograms, 6 for each phototransformation experiment-Figure S20) recorded in TOF (MS) mode were aligned with MPP software giving 179 entities. After a build-in MPP filtration by flags filtering, abundance $(5 \%-80 \%)$ and Kruskal-Wallis test ( $p=0.0 .1$ cut-off) 52 entities were finally selected for the chemometric study.

The principal component analysis (PCA) based on this data is shown in Figure 8. The PCA is a chemometric technique, widely used for the exploratory data analysis and samples relationships visualization. Its principle relies on the conversion of the original variables to the equal number of latent variables (principal components), which are orthogonal, and explain the largest percent of the data variance. The first three principal components explained $79.08 \%$ of the total variance. The distribution of the samples (spheres of each color represent each experiment) shows that $\mathrm{TiO}_{2}$-catalyzed samples (turquoise spheres) were the most distant (and therefore the least similar) from the standard samples (sertindole working solution before the experiment, gray spheres). Mixed catalysts samples were placed between these extreme conditions. No significant differences between $\mathrm{TiO}_{2}-\mathrm{Fe}_{2} \mathrm{O}_{3}$ (dark red spheres) and $\mathrm{TiO}_{2}-\mathrm{FeOOH}$ (dark blue spheres) were observed, however, $\mathrm{TiO}_{2}-\mathrm{Fe}_{2} \mathrm{O}_{3}$ seem to be a little closer to the remaining samples. The direct photolysis (brown spheres), $\mathrm{Fe}_{2} \mathrm{O}_{3}$ (red spheres), $\mathrm{FeOOH}$ (blue spheres) photocatalysis, and pharmaceutical formulations (pink spheres for $\mathrm{S} 4$ and green spheres for S16) samples were distributed along the Z-axis. As it can be seen, FeOOH samples were the most similar to the direct photolysis which should be explained by its low catalytic activity. Nevertheless, despite the sertindole decomposition in $\mathrm{FeOOH}$-catalyzed experiment was slower than 
during the direct photolysis, in the TPs profile the effects of photocatalysis are pronounced. Such an observation indicates that the presence of this compound as a pigment could affect the formation of the transformation products. $\mathrm{Fe}_{2} \mathrm{O}_{3}$ samples formed a more outlying group which could be explained by a higher activity of this catalyst. The relationships between $\mathrm{FeOOH}$ and $\mathrm{Fe}_{2} \mathrm{O}_{3}$ resemble the relationships between S4 and S16 pharmaceutical formulations-which reflects the influence of the applied pigments on the studied process. Generally, it should be noted that the presence of $\mathrm{FeOOH}$ and $\mathrm{Fe}_{2} \mathrm{O}_{3}$ as pigments could affect (however not to a large extent) the phototransformation processes and it should be taken into account during the analysis of the pharmaceutical formulations.

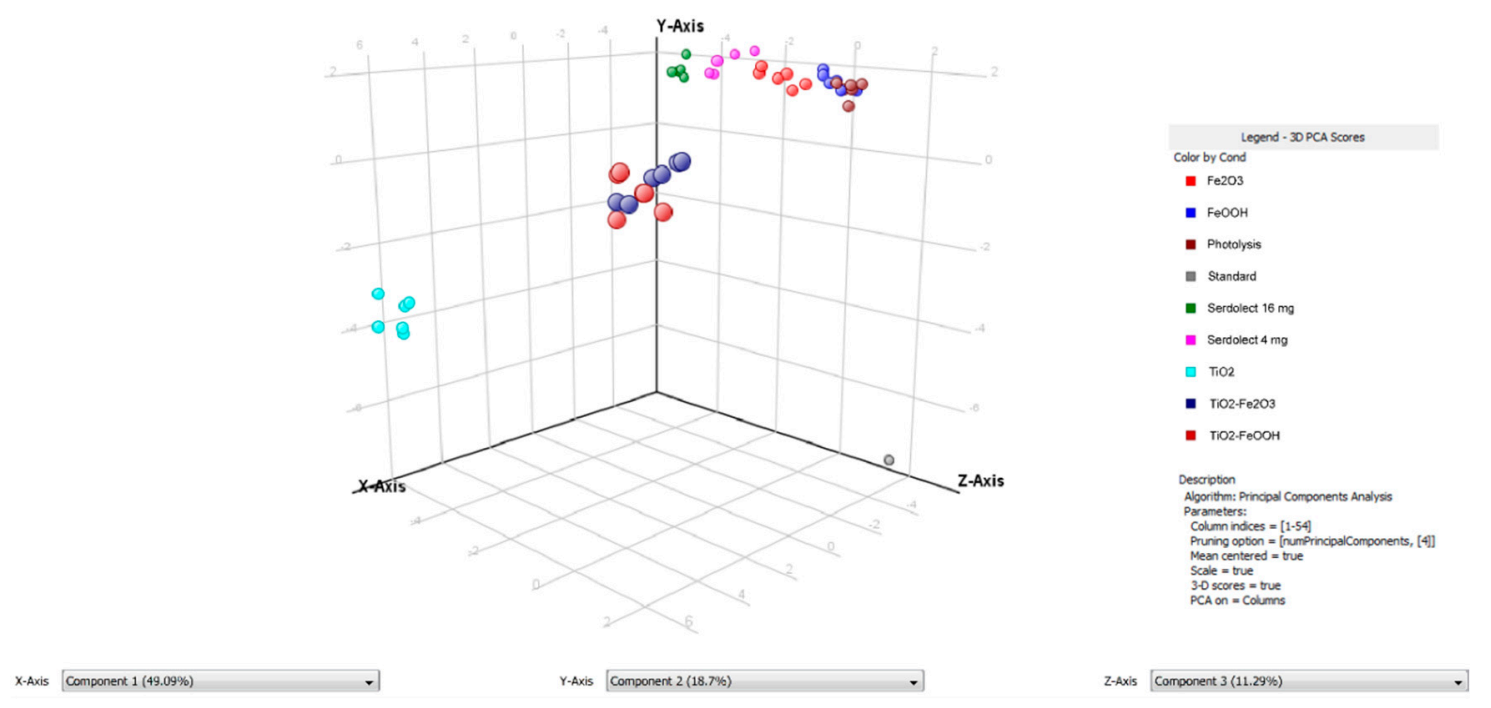

Figure 8. $3 \mathrm{D}$ principal component analysis (PCA) plot of irradiated and non-irradiated sertindole samples.

\subsection{In Silico Evaluation of TPs Properties}

In this study the acute toxicity to rodents and mutagenicity of sertindole TPs as well as the parent compound are studied. Additionally, since sertindole is a well-known potassium channel blocker, a probability of hERG inhibition was also calculated. In the case of toxicity to rodents, where six models were applied, PCA was performed. This multivariate exploratory chemometric technique enables the reduction of dimensionality of the data matrices and visualization of relationships between compounds as well as between models.

\subsubsection{Acute Toxicity to Rodents}

In silico toxicity to rodents of sertindole as well as its TPs were assessed with the use of ACD/Percepta 14.0.0 (ACD/Labs, 2015 Release). Six toxicity categories were applied: two for rats—oral (OR) and intraperitoneal (IP) and four for mice—oral (OR), intravenous (IV), subcutaneous (SC), intraperitoneal (IP). The calculated $\mathrm{LD}_{50}$ values (expressed in $\log _{\mathrm{mg} \mathrm{kg}}{ }^{-1}$ ) are presented in Table S3. In order to visualize the relationships between the toxicity of the analyzed compounds, a principal component analysis was performed. The first principal component (PC1) explained $65.85 \%$ of the total variability and the second principal component (PC2)-19.78\%. As shown in Figure 9 (toxicity decreases in parallel with increasing $\mathrm{LD}_{50}$ values), TP1, TP6, TP14-TP18, and sertindole possessed very similar toxicity to rodents. TP4, TP8, TP9, TP12, and TP13 were generally less toxic for all the models (in the case of TP8 - with the exception of the rat oral model which predicted almost the same toxicity). TP3, TP11, and especially TP2 were less toxic for the rat models and possessed very similar toxicity to mice. TP7 should be considered as the least toxic amongst the studied compounds, especially to 
rats. TP5 and TP10 were more toxic to mice than the parent compound, however, on the other hand, their toxicity to rats was significantly lower.

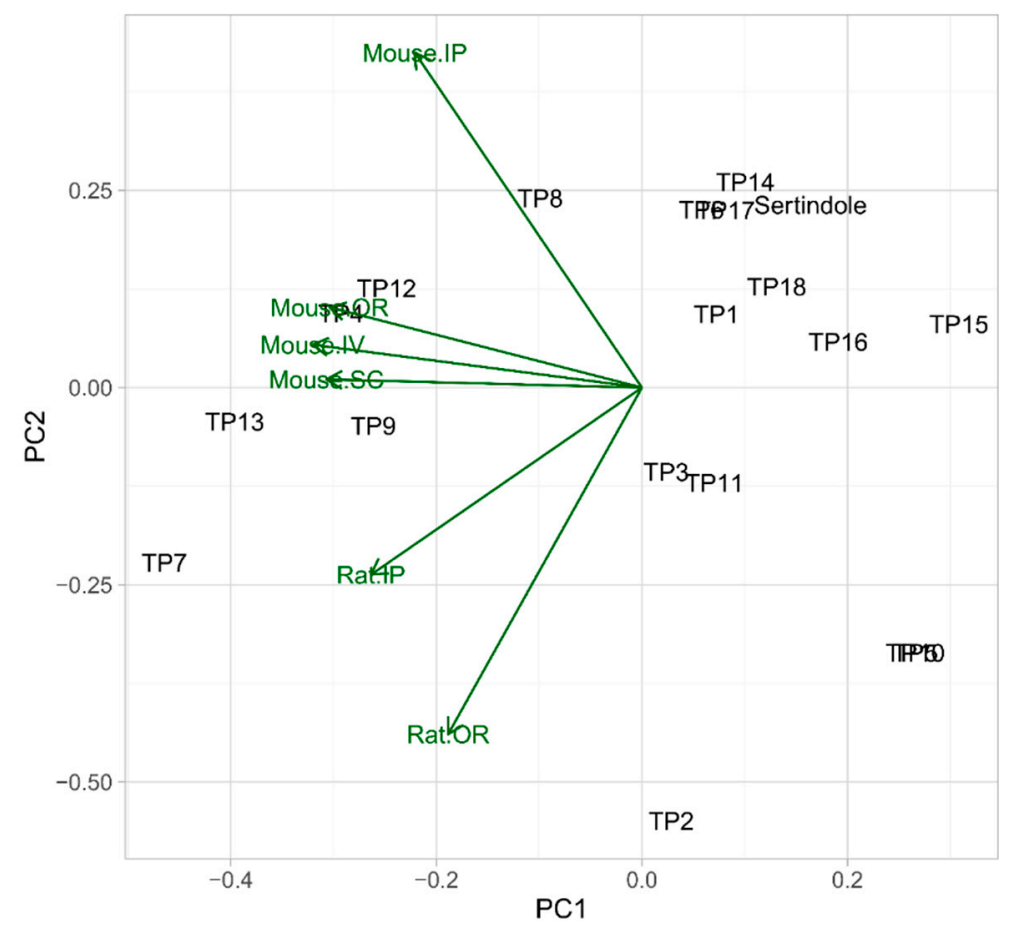

Figure 9. Comparison of acute toxicity to rodents of sertindole and its TPs by PCA.

\subsubsection{Mutagenicity}

The mutagenicity of sertindole and its TPs expressed as the probability of a positive result of a commonly applied Ames test is shown in Table 4. The majority of the TPs possessed higher predicted mutagenicity, however, only in the case of TP2 and TP15-TP17 the probability exceeded 0.5. Such findings suggest that the introduction of two double bonds into the piperidine ring of the sertindole molecule significantly increases the mutagenicity of the compound. Moreover, as TP2 belongs to the major photodegradation products, the interaction of sertindole with UV-Vis radiation could result in the higher mutagenic potential. 
Table 4. Mutagenicity and hERG inhibitory potential of sertindole and its TPs.

\begin{tabular}{ccc}
\hline Compound & Mutagenicity & hERG inhibition \\
\hline Sertindole & 0.39 & 0.95 \\
TP1 & 0.4 & 0.71 \\
TP2 & 0.58 & 0.33 \\
TP3 & 0.4 & 0.57 \\
TP4 & 0.4 & 0.37 \\
TP5 & 0.48 & 0.79 \\
TP6 & 0.38 & 0.87 \\
TP7 & 0.41 & 0.06 \\
TP8 & 0.26 & 0.92 \\
TP9 & 0.25 & 0.68 \\
TP10 & 0.48 & 0.79 \\
TP11 & 0.26 & 0.75 \\
TP12 & 0.26 & 0.72 \\
TP13 & 0.36 & 0.21 \\
TP14 & 0.37 & 0.88 \\
TP15 & 0.78 & 0.53 \\
TP16 & 0.84 & 0.37 \\
TP17 & 0.7 & 0.4 \\
TP18 & 0.45 & 0.92 \\
\hline
\end{tabular}

\subsection{3. hERG Inhibition}

Sertindole is a well-known blocker of hERG potassium channel, which could result in the prolongation of QT interval on the electrocardiogram [9]. Therefore, hERG inhibitory potential of the sertindole TPs was also evaluated. The applied model has correctly predicted the parent compound properties-the calculated probability of inhibition $\left(\mathrm{K}_{\mathrm{i}}<10 \mu \mathrm{M}\right)$ was 0.95 , which was the highest value among the studied compounds. Very similar values were obtained for TP8 and TP18 which suggest that neither dechlorination nor hydroxylation of an indole moiety leads to the decrease of hERG inhibitory potential. On the other hand, double N-oxidation (TP2), the removal of a 5-fluorophenyl moiety (TP9) or dehydrogenation of a piperidine ring (TP4, TP13 and TP15-TP17) significantly reduces the probability of hERG inhibition.

\section{Conclusions}

In this study the photostability of sertindole under the simulated solar radiation was evaluated. On the contrary to the available literature, the studied compound turned out to be highly photolabile-after $16 \mathrm{~min}$ of irradiation more than $75 \%$ of the initial amount was decomposed. Amongst the formed TPs the major one was a product of sertindole dechlorination followed by hydroxylation. The remaining TPs-the products of aromatic and aliphatic hydroxylation, oxidation, $\mathrm{N}$-oxidation, dehydrogenation, and dehalogenation—were significantly less abundant.

Additionally, the influence of three metal oxides, commonly used as pigments in pharmaceutical formulations, on the qualitative and quantitative aspect of the phototransformation process was studied. $\mathrm{TiO}_{2}$ influenced the process to the largest extent and after $16 \mathrm{~min}$ sertindole was practically undetectable. The impact of two iron oxides, $\mathrm{FeOOH}$ and $\mathrm{Fe}_{2} \mathrm{O}_{3}$ was significantly lower-FeOOH slightly inhibited the degradation while $\mathrm{Fe}_{2} \mathrm{O}_{3}$ accelerated the process. The application of the mixtures containing $\mathrm{TiO}_{2}$ and iron oxides gave the analogous results - in both cases an increase in the degradation rate was observed, however, in the case of $\mathrm{TiO}_{2}-\mathrm{FeOOH}$ mixture to a less extent. The obtained results were compared with the experiments using the real pharmaceutical formulations-Serdolect ${ }^{\circledR} 4 \mathrm{mg}$ and Serdolect ${ }^{\circledR} 16 \mathrm{mg}$, containing $\mathrm{TiO}_{2}$ with $\mathrm{FeOOH}$ and $\mathrm{TiO}_{2}$ with $\mathrm{Fe}_{2} \mathrm{O}_{3}$, respectively, as the excipients. The degradation rates were very similar in both cases and higher than in the direct photolysis, which should be attributed to the activity of $\mathrm{TiO}_{2}$. The exploration of the results with use of PCA revealed close relationship between the profiles obtained from $\mathrm{FeOOH}, \mathrm{Fe}_{2} \mathrm{O}_{3}$, and the pharmaceutical 
formulations samples, which indicates that the application of iron oxides as pigments could affect both a quantitative and qualitative aspect of the phototransformation process. From this point of view the other metal oxides characterized by lower photocatalytic activity should be considered as pigments used in pharmaceutical formulations, especially in the case of titanium oxide.

In silico assessment of toxicity showed that the majority of the TPs are less toxic to rodents than the parent compound. On the other hand, several products, including relatively abundant TP2, could possess a significant mutagenic potential. Most of the TPs were marked by high hERG inhibitory potential, however, none of the products surpassed sertindole in this regard.

Supplementary Materials: The following are available online at http://www.mdpi.com/1999-4923/11/7/299/s1, Figure S1: MS/MS spectrum and fragmentation pattern of sertindole, Figure S2: MS/MS spectrum and fragmentation pattern of TP1, Figure S3: MS/MS spectrum and fragmentation pattern of TP2, Figure S4: MS/MS spectrum and fragmentation pattern of TP3, Figure S5: MS/MS spectrum and fragmentation pattern of TP4, Figure S6: MS/MS spectrum and fragmentation pattern of TP5, Figure S7: MS/MS spectrum and fragmentation pattern of TP6, Figure S8: MS/MS spectrum and fragmentation pattern of TP7, Figure S9: MS/MS spectrum and fragmentation pattern of TP8, Figure S10: MS/MS spectrum and fragmentation pattern of TP9, Figure S11: MS/MS spectrum and fragmentation pattern of TP10, Figure S12: MS/MS spectrum and fragmentation pattern of TP11, Figure S13: MS/MS spectrum and fragmentation pattern of TP12, Figure S14: MS/MS spectrum and fragmentation pattern of TP13, Figure S15: MS/MS spectrum and fragmentation pattern of TP14, Figure S16: MS/MS spectrum and fragmentation pattern of TP15, Figure S17: MS/MS spectrum and fragmentation pattern of TP16, Figure S18:, Figure S19: MS/MS spectrum and fragmentation pattern of TP18, Figure S20: Total ion chromatograms (overlay) of irradiated sertindole samples, Table S1: Calibration of the UHPLC method with DAD detection for determination of sertindole, Table S2: Robustness of the UHPLC method with DAD detection for determination of sertindole, Table S3: Toxicity of sertindole and its TPs to rodents.

Author Contributions: Conceptualization, R.S.; methodology, R.S.; formal analysis, J.T.; investigation J.T. and R.S.; data curation, J.T. and R.S.; writing original draft, J.T.; writing-editing and review, R.S.; supervision, R.S.

Funding: This research received no external funding.

Conflicts of Interest: The authors declare no conflict of interest.

\section{References}

1. Tonnesen, H.H. Photostability of Drugs and Drug Formulations, 2nd ed.; CRC Press: Boca Raton, FL, USA, 2004; ISBN 978-1-4200-2359-6.

2. Trawiński, J.; Skibiński, R. Studies on photodegradation process of psychotropic drugs: A review. Environ. Sci. Pollut. Res. 2017, 24, 1152-1199. [CrossRef] [PubMed]

3. International Council for Harmonization of Technical Requirements for Pharmaceuticals for Human Use. Stability Testing: Photostability Testing of New Drug Substances and Products: - Q1B_Guideline.pdf. Available online: http://www.ich.org/fileadmin/Public_Web_Site/ICH_Products/Guidelines/Quality/Q1B/ Step4/Q1B_Guideline.pdf (accessed on 7 July 2016).

4. Di Paola, A.; García-López, E.; Marcì, G.; Palmisano, L. A survey of photocatalytic materials for environmental remediation. J. Hazard. Mater. 2012, 211-212, 3-29. [CrossRef] [PubMed]

5. Kakinoki, K.; Yamane, K.; Igarashi, M.; Yamamoto, M.; Teraoka, R.; Matsuda, Y. Evaluation of titanium dioxide as a pharmaceutical excipient for preformulation of a photo-labile drug: Effect of physicochemical properties on the photostability of solid-state nisoldipine. Chem. Pharm. Bull. (Tokyo) 2005, 53, 811-815. [CrossRef] [PubMed]

6. Hyttel, J.; Nielsen, J.B.; Nowak, G. The acute effect of sertindole on brain 5-HT $\mathrm{H}_{2}$ and $\alpha_{1}$ receptors (ex vivo radioreceptor binding studies). J. Neural Transm. 1992, 89, 61-69. [CrossRef]

7. Sánchez, C.; Arnt, J.; Dragsted, N.; Hyttel, J.; Lembøl, H.L.; Meier, E.; Perregaard, J.; Skarsfeldt, T. Neurochemical and in vivo pharmacological profile of sertindole, a limbic-selective neuroleptic compound. Drug Dev. Res. 1991, 22, 239-250. [CrossRef]

8. Van Kammen, D.P.; McEvoy, J.P.; Targum, S.D.; Kardatzke, D.; Sebree, T.B. A randomized, controlled, dose-ranging trial of sertindole in patients with schizophrenia. Psychopharmacology (Berl.) 1996, 124, 168-175. [CrossRef] [PubMed]

9. Moore, N.A. Behavioural pharmacology of the new generation of antipsychotic agents. Br. J. Psychiatry 1999, 174, 5-11. [CrossRef] 
10. Canal-Raffin, M.; Déridet, E.; Titier, K.; Frakra, E.; Molimard, M.; Moore, N. Simplified ultraviolet liquid chromatographic method for determination of sertindole, dehydrosertindole and norsertindole, in human plasma. J. Chromatogr. B 2005, 814, 61-67. [CrossRef] [PubMed]

11. El-Kosasy, A.M.; Hussein, L.A.; Sedki, N.G.; Salama, N.N. Micelle enhanced and native spectrofluorimetric methods for determination of sertindole using sodium dodecyl sulfate as sensitizing agent. Spectrochim. Acta. A. Mol. Biomol. Spectrosc. 2016, 153, 422-427. [CrossRef] [PubMed]

12. Tzeng, T.-B.; Stamm, G.; Chu, S. Sensitive method for the assay of sertindole in plasma by high-performance liquid chromatography and fluorimetric detection. J. Chromatogr. B. Biomed. Sci. Appl. 1994, 661, 299-306. [CrossRef]

13. Choong, E.; Rudaz, S.; Kottelat, A.; Guillarme, D.; Veuthey, J.-L.; Eap, C.B. Therapeutic drug monitoring of seven psychotropic drugs and four metabolites in human plasma by HPLC-MS. J. Pharm. Biomed. Anal. 2009, 50, 1000-1008. [CrossRef] [PubMed]

14. El-Ragehy, N.A.; Hassan, N.Y.; Abdelkawy, M.; Tantawy, M.A. Stability-indicating chromatographic methods for the determination of sertindole. J. Chromatogr. Sci. 2014, 52, 559-565. [CrossRef] [PubMed]

15. Trawiński, J.; Skibiński, R.; Komsta, Ł. Comparison of ESI and APCI sources in Q-TOF mass spectrometer in photodegradation study of selected psychotropic drugs. Acta Chromatogr. 2017, 29, 161-172. [CrossRef]

16. Bandara, J.; Mielczarski, J.A.; Lopez, A.; Kiwi, J. 2. Sensitized degradation of chlorophenols on iron oxides induced by visible light comparison with titanium oxide. Appl. Catal. B Environ. 2001,34,321-333. [CrossRef]

(C) 2019 by the authors. Licensee MDPI, Basel, Switzerland. This article is an open access article distributed under the terms and conditions of the Creative Commons Attribution (CC BY) license (http://creativecommons.org/licenses/by/4.0/). 